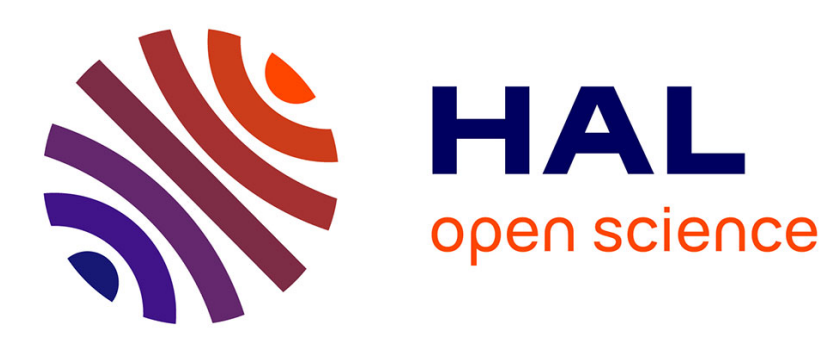

\title{
Homogénéisation par la méthode des formes moyennes équivalentes dans le milieu biphasé
}

Jacques Grolier, Monique Hucher, Jean-Marie Pouliquen, Joëlle Riss, Michel Lavaud

\section{- To cite this version:}

Jacques Grolier, Monique Hucher, Jean-Marie Pouliquen, Joëlle Riss, Michel Lavaud. Homogénéisation par la méthode des formes moyennes équivalentes dans le milieu biphasé. Revue de Physique Appliquée, 1989, 24 (6), pp.627-642. 10.1051/rphysap:01989002406062700 . jpa-00246088

\section{HAL Id: jpa-00246088 https://hal.science/jpa-00246088}

Submitted on 1 Jan 1989

HAL is a multi-disciplinary open access archive for the deposit and dissemination of scientific research documents, whether they are published or not. The documents may come from teaching and research institutions in France or abroad, or from public or private research centers.
L'archive ouverte pluridisciplinaire HAL, est destinée au dépôt et à la diffusion de documents scientifiques de niveau recherche, publiés ou non, émanant des établissements d'enseignement et de recherche français ou étrangers, des laboratoires publics ou privés. 


\title{
Homogénéisation par la méthode des formes moyennes équivalentes dans le milieu biphasé
}

\author{
Jacques Grolier $\left(^{1}\right)$, Monique Hucher $\left(^{1}\right)$, Jean-Marie Pouliquen $\left(^{1}\right.$ ), Joëlle Riss $\left({ }^{1}\right)$ \\ et Michel Lavaud ( ${ }^{2}$ ) \\ (1) Université d'Orléans, BP 6759, 45067 Orléans, France \\ (2) G.R.E.M.I., C.N.R.S., Orléans, France
}

(Reçu le 27 avril 1988, révisé le 16 janvier 1989, accepté le 27 février 1989)

\begin{abstract}
Résumé. - On peut calculer les conductivités (thermique, électrique, etc.) des roches à partir des conductivités et des fractions volumiques des minéraux constitutifs, de la forme des grains représentée par un «ellipsoïde moyen équivalent » (E.M.E.), et de leur orientation moyenne. La théorie présentée étend aux ellipsoïdes quelconques la théorie d'Ondracek jusqu'ici limitée aux ellipsoïdes de révolution.
\end{abstract}

\begin{abstract}
A theory is presented, extending Ondracek's derivation which is normally restricted to oblate and prolate spheroids, to the case of any kind of ellipsoid representing the texture of the aggregate as an average equivalent shape (A.E.S.). With such a theory, the effective conductivity of any rock can be calculated from the conductivities of rock forming minerals, from the volume fractions, the lengths and orientations of the semi-axes $a, b, c$ of the average equivalent ellipsoid determined by quantitative microscopy.
\end{abstract}

Notations.

\section{Première partie : Généralisation de la théorie d'Ondracek.}

$\mathrm{O} X, \mathrm{O} Y, \mathrm{OZ}$ : axes principaux de l'ellipsoïde.

$a, b, c \quad:$ longueurs des demi-axes principaux de l'ellipsoïde.

$\alpha_{X \mathrm{D}}, \alpha_{Y \mathrm{D}}, \alpha_{Z \mathrm{D}}$ : angles entre les axes principaux de l'ellipsoïde et la direction du champ.

\section{Electrostatique.}

$\mathbf{E}_{\mathrm{M}}, E_{\mathrm{M}} \quad$ : champ et intensité du champ dans la matrice.

$\mathbf{E}_{\mathrm{D}}, E_{\mathrm{D}} \quad$ : champ et intensité du champ à l'intérieur d'un grain (de forme ellipsoïdale) de la phase dispersée.

$\mathbf{E}_{X}, \mathbf{E}_{Y}, \mathbf{E}_{Z} \quad$ : champs respectivement parallèles aux axes $\mathrm{O} X, \mathrm{O} Y, \mathrm{OZ}$ de l'ellipsoïde.

$E_{\mathrm{D} X}, E_{\mathrm{D} Y}, E_{\mathrm{DZ}}$ : composantes du champ suivant chacun des axes de l'ellipsoïde. $\varepsilon_{\mathrm{c}}, \varepsilon_{\mathrm{M}}, \varepsilon_{\mathrm{D}}:$ constantes diélectriques $\mathrm{du}$ composite, de la matrice, de la phase dispersée.

$F_{X \mathrm{D}}, F_{\mathrm{YD}}, F_{\mathrm{ZD}}$ : coefficients de dépolarisation.

$D_{\mathrm{c}}, D_{\mathrm{M}}, D_{\mathrm{D}} \quad$ : induction électrique du composite biphasé, de la matrice, de la phase dispersée.

GÉNÉRALISATION AUX AUTRES PROPRIÉTÉS PHYSIQUES.

$\varphi_{\mathrm{c}}, \varphi_{\mathrm{M}}, \varphi_{\mathrm{D}} \quad$ : propriétés de champ du composite, de la matrice, de la phase dispersée.

$c_{\mathrm{D}} \quad:$ fraction volumique de la phase dispersée.

$c_{\mathrm{M}} \quad:$ fraction volumique de la matrice $\left(c_{\mathrm{D}}+c_{\mathrm{M}}=1\right)$.

DÉCOMPOSITION DE L'ÉQUATION DIFFÉRENTIELLE (17) EN ÉLÉMENTS SIMPLES.

$\lambda=F_{\mathrm{XD}} ; \mu=F_{\mathrm{YD}} ; \nu=F_{\mathrm{ZD}} ; t=\varphi_{\mathrm{c}} / \varphi_{\mathrm{D}} ; t_{\mathrm{n}}=$ $\varphi_{\mathrm{M}} / \varphi_{\mathrm{D}}$

$\ell=\cos ^{2} \alpha_{X \mathrm{D}} ; \quad m=\cos ^{2} \alpha_{Y \mathrm{D}} ; \quad n=\cos ^{2} \alpha_{Z \mathrm{D}}$ $x=c_{\mathrm{D}}$. 
Deuxième partie : Prise en compte des données de la microscopie.

$A_{1}, A_{2}, A_{3}:$ axes principaux de l'ellipsoïde.

$\overline{\bar{A}}, \overline{\bar{B}}, \overline{\bar{H}}, \overline{\bar{L}}, \overline{\overline{L^{4}}}, N_{\mathrm{A}}, N_{\mathrm{L}}, N_{\mathrm{V}}, \bar{S}, \bar{V}$ : cf. équation (93).

$a^{\prime}, b^{\prime}, a^{\prime \prime}, b^{\prime \prime}, a^{\prime \prime \prime}, b^{\prime \prime \prime}:$ longueurs des demi-axes principaux des ellipses.

$\theta_{1}, \theta_{2}, \theta_{3}$ : angles entre les axes principaux de l'ellipsoïde et l'axe $\mathrm{O} X_{1}$.

$\gamma_{1}, \gamma_{2}, \gamma_{3}$ : angles entre les axes principaux de l'ellipsoïde et l'axe $\mathrm{O} X_{2}$.

$\alpha_{1}, \alpha_{2}, \alpha_{3}:$ angles entre les axes principaux de l'ellipsoïde et l'axe $\mathrm{O} X_{3}$.

$\beta^{\prime} \quad$ : angle entre le plus grand des axes principaux de l'ellipse (coupe diamétrale de l'ellipsoïde par le plan $\mathrm{O} X_{2} X_{3}$ ) et $\mathrm{O} X_{3}$.

$\beta^{\prime \prime} \quad$ : angle entre le plus grand des axes principaux de l'ellipse (coupe diamétrale de l'ellipsoïde par le plan $\left.\mathrm{O} X_{1} X_{3}\right)$ et $\mathrm{O} X_{3}$.

$\beta^{\prime \prime \prime} \quad$ : angle entre le plus grand des axes principaux de l'ellipse (coupe diamétrale de l'ellipsoïde par le plan $\left.\mathrm{O} X_{1} X_{2}\right)$ et $\mathrm{O} X_{2}$

La conductivité thermique, ou électrique, la perméabilité magnétique et plus généralement toute propriété de champ (c'est-à-dire toute grandeur physique mesurant la réponse d'un corps soumis à un champ uniforme) d'un milieu polyphasé, dépendent non seulement des propriétés physiques de ses constituants et de leurs fractions volumiques, mais aussi de la forme des particules et de l'orientation de l'échantillon, lorsque ce dernier est anisotrope, par rapport au champ appliqué. Si l'on ne tient compte que des conductivités et des fractions volumiques, on détermine seulement un intervalle de valeurs limité par les bornes de Hashin et Shrikman (19621968) entre lesquelles est située la conductivité globale ou conductivité effective du milieu considéré.

L'une des théories permettant de prendre en compte la forme des particules et l'anisotropie de l'échantillon a été développée par Ondracek (19821983 et travaux cités) et ses collaborateurs dans le cadre de la métallurgie physique des matériaux frittés (Pejsa, 1980 ; Klein et Ondracek, 1981) et du traitement des minerais de fer (Ebel, 1984 ; Ebel et al., 1985). On s'inspire ici de ces travaux en en explicitant certains aspects et, en généralisant la méthode, on montre comment utiliser les données de la microscopie optique, pour obtenir des valeurs précises et exactes de la conductivité effective.
1. Généralisation de la théorie d'Ondracek (cas de l'ellipsoïde quelconque).

Le point de départ de la théorie d'Ondracek (1982) est un résultat de l'électrostatique donnant l'intensité du champ $\mathbf{E}_{\mathrm{D}}$ à l'intérieur d'un ellipsoïde de constante diélectrique $\varepsilon_{\mathrm{D}}$. Si celui-ci est plongé dans un milieu de constante diélectrique $\varepsilon_{M}$ où règne un champ $\mathbf{E}_{\mathrm{M}}$, et si l'un des axes de l'ellipsoïde (ici l'axe $\mathrm{OX}$ ) est parallèle à $\mathbf{E}_{\mathrm{M}}$,

$$
\frac{E_{\mathrm{D}}}{E_{\mathrm{M}}}=\frac{\varepsilon_{\mathrm{M}}}{\varepsilon_{\mathrm{M}}+\left(\varepsilon_{\mathrm{D}}-\varepsilon_{\mathrm{M}}\right) F_{X \mathrm{D}}}
$$

où les indices $\mathrm{D}$ et $\mathrm{M}$ représentent respectivement la phase dispersée (particules) et la matrice ; $F_{X \mathrm{D}}$ est un coefficient dit de dépolarisation ; avec des équations analogues lorsque les axes $\mathrm{OY}$ et $\mathrm{OZ}$ de l'ellipsoïde sont parallèles à $\mathbf{E}_{\mathbf{M}}$. Les coefficients de dépolarisation sont donnés par les équations de Stoner (1945, Eqs. (3-4)) ; avec

$$
F_{X \mathrm{D}}+F_{Y \mathrm{D}}+F_{Z \mathrm{D}}=1 \text {. }
$$

Si les $a, b, c$ sont les demi-axes de l'ellipsoïde moyen équivalent (E.M.E.) (Riss et Grolier, 1986) déterminé par l'étude stéréologique du solide réel considéré, les valeurs numériques des $F_{i \mathrm{D}}$ obtenues en portant $a, b, c$, dans les formules de Stoner sont significatives de la forme des grains dans ce solide et du comportement de celui-ci quand il est placé dans un champ.

Soient $\alpha_{X \mathrm{D}}, \alpha_{Y \mathrm{D}}, \alpha_{Z \mathrm{D}}$, les angles entre la direction $E_{\mathrm{M}}$ du champ dans la matrice et chacun des axes principaux de l'E.M.E. On peut écrire, suivant (1) :

$$
\begin{aligned}
& E_{\mathrm{D} X}=\frac{\varepsilon_{\mathrm{M}} E_{\mathrm{M}} \cos \alpha_{X \mathrm{D}}}{\varepsilon_{\mathrm{M}}+\left(\varepsilon_{\mathrm{D}}-\varepsilon_{\mathrm{M}}\right) F_{X \mathrm{D}}} ; \\
& E_{\mathrm{D} Y}=\frac{\varepsilon_{\mathrm{M}} E_{\mathrm{M}} \cos \alpha_{\mathrm{YD}}}{\varepsilon_{\mathrm{M}}+\left(\varepsilon_{\mathrm{D}}-\varepsilon_{\mathrm{M}}\right) F_{\mathrm{YD}}} ; \\
& E_{\mathrm{DZ}}=\frac{\varepsilon_{\mathrm{M}} E_{\mathrm{M}} \cos \alpha_{\mathrm{ZD}}}{\varepsilon_{\mathrm{M}}+\left(\varepsilon_{\mathrm{D}}-\varepsilon_{\mathrm{M}}\right) F_{\mathrm{ZD}}} .
\end{aligned}
$$

La composante de $\mathbf{E}_{\mathrm{D}}$ suivant la direction $\mathbf{E}_{\mathbf{M}}$ est la somme des champs apparents d'intensité : $E_{\mathrm{D} X} \cos \alpha_{\mathrm{D} X}, E_{\mathrm{D} Y} \cos \alpha_{\mathrm{DY}}, E_{\mathrm{DZ}} \cos \alpha_{\mathrm{DZ}}$, son intensité est donc:

$$
E_{\mathrm{D}}=E_{\mathrm{M}} \sum_{i=X}^{z} \frac{\varepsilon_{\mathrm{M}} \cos ^{2} \alpha_{i \mathrm{D}}}{\varepsilon_{\mathrm{M}}+\left(\varepsilon_{\mathrm{D}}-\varepsilon_{\mathrm{M}}\right) F_{i \mathrm{D}}}
$$

L'intensité du champ $E_{\mathrm{c}}$ d'un biphasé et celle de son induction électrique $D_{\mathrm{c}}$ peuvent s'exprimer en fonction des grandeurs $E_{\mathrm{M}}, E_{\mathrm{D}}$ et $D_{\mathrm{M}}, D_{\mathrm{D}}$ relatives aux deux phases à l'aide des moyennes de Wiener :

$$
E_{\mathrm{c}}=c_{\mathrm{M}} E_{\mathrm{M}}+c_{\mathrm{D}} E_{\mathrm{D}} \text { et } D_{\mathrm{c}}=c_{\mathrm{M}} D_{\mathrm{M}}+c_{\mathrm{D}} D_{\mathrm{D}}
$$


où $c_{\mathrm{D}}$ est la fraction volumique de la phase incluse et $c_{M}=1-c_{D}$, la fraction volumique de la matrice, avec :

$$
D_{\mathrm{c}}=\varepsilon_{\mathrm{c}} E_{\mathrm{c}} ; \quad D_{\mathrm{M}}=\varepsilon_{\mathrm{M}} E_{\mathrm{M}} ; \quad D_{\mathrm{D}}=\varepsilon_{\mathrm{D}} E_{\mathrm{D}}
$$

La polarisation étant en effet un phénomène intéressant tout le volume, les grandeurs qui y interviennent sont des grandeurs extensives. Par suite :

$$
\frac{E_{\mathrm{D}}}{E_{\mathrm{M}}}=\frac{c_{\mathrm{M}}}{c_{\mathrm{D}}} \cdot \frac{\varepsilon_{\mathrm{M}}-\varepsilon_{\mathrm{c}}}{\varepsilon_{\mathrm{c}}-\varepsilon_{\mathrm{D}}} .
$$

Comparant à l'équation générale (4), on obtient :

$$
\begin{aligned}
& \frac{c_{M}}{c_{\mathrm{D}}} \cdot \frac{\varepsilon_{\mathrm{M}}-\varepsilon_{\mathrm{c}}}{\varepsilon_{\mathrm{c}}-\varepsilon_{\mathrm{D}}}=\varepsilon_{\mathrm{M}}\left[\frac{\cos ^{2} \alpha_{X \mathrm{D}}}{\varepsilon_{\mathrm{M}}+\left(\varepsilon_{\mathrm{D}}-\varepsilon_{\mathrm{M}}\right) F_{X \mathrm{D}}}+\right. \\
& \left.\quad+\frac{\cos ^{2} \alpha_{Y \mathrm{D}}}{\varepsilon_{\mathrm{M}}+\left(\varepsilon_{\mathrm{D}}-\varepsilon_{\mathrm{M}}\right) F_{\mathrm{ID}}}+\frac{\cos ^{2} \alpha_{Z \mathrm{D}}}{\varepsilon_{\mathrm{M}}+\left(\varepsilon_{\mathrm{D}}-\varepsilon_{\mathrm{M}}\right) F_{Z \mathrm{D}}}\right] .
\end{aligned}
$$

Comme Ondracek, nous remplaçons ici les $\varepsilon$ par $\operatorname{des} \varphi$

$$
\varepsilon_{\mathrm{c}} \rightarrow \varphi_{\mathrm{c}}, \quad \varepsilon_{\mathrm{M}} \rightarrow \varphi_{\mathrm{M}}, \quad \varepsilon_{\mathrm{D}} \rightarrow \varphi_{\mathrm{D}}
$$

pour signifier que l'équation (7), et par suite la théorie établie en électrostatique, vaut en fait pour toute propriété de champ :

$$
\begin{aligned}
\frac{c_{\mathrm{M}}}{c_{\mathrm{D}}} \cdot \frac{\varphi_{\mathrm{M}}-\varphi_{\mathrm{c}}}{\varphi_{\mathrm{c}}-\varphi_{\mathrm{D}}} & =\varphi_{\mathrm{M}} \sum_{i=X}^{z} \frac{\cos ^{2} \alpha_{i \mathrm{D}}}{\varphi_{\mathrm{M}}+\left(\varphi_{\mathrm{D}}-\varphi_{\mathrm{M}}\right) F_{i \mathrm{D}}} \\
& =\varphi_{\mathrm{M}} \Omega
\end{aligned}
$$

d'où l'on tire :

$$
\varphi_{c}=\varphi_{M} \frac{c_{M}+c_{D} \varphi_{D} \Omega}{c_{M}+c_{D} \varphi_{M} \Omega}
$$

Lorsque l'ellipsoïde est de révolution, $F_{X \mathrm{D}}=F_{Y \mathrm{D}}$ Posons $F_{X \mathrm{D}}=F_{\mathrm{YD}}=F_{\mathrm{D}}$ et donc, $F_{Z \mathrm{D}}=1-2 F_{\mathrm{D}}$. Par ailleurs, $\cos ^{2} \alpha_{X \mathrm{D}}=\cos ^{2} \alpha_{Y \mathrm{D}}$; posons $\cos ^{2} \alpha_{Z D}=\cos ^{2} \alpha_{D}$. L'équation (9) devient alors, en remplaçant $c_{\mathrm{M}}$ par $1-c_{\mathrm{D}}$ :

$$
\left.\varphi_{c}=\varphi_{M} \frac{1-c_{D}+c_{D} \varphi_{D}\left\{\frac{1-\cos ^{2} \alpha_{D}}{\varphi_{M}+\left(\varphi_{D}-\varphi_{M}\right) F_{D}}+\frac{\cos ^{2} \alpha_{D}}{\varphi_{M}+\left(\varphi_{D}-\varphi_{M}\right)\left(1-2 F_{D}\right)}\right.}{1-c_{D}+c_{D} \varphi_{M}\left\{\frac{1-\cos ^{2} \alpha_{D}}{\varphi_{M}+\left(\varphi_{D}-\varphi_{M}\right) F_{D}}+\frac{\cos ^{2} \alpha_{D}}{\varphi_{M}+\left(\varphi_{D}-\varphi_{M}\right)\left(1-2 F_{D}\right)}\right.}\right\}
$$

analogue à l'équation (19b) d'Ondracek (1983).

Lorsque l'ellipsoïde se réduit à une sphère, on a : $F_{X \mathrm{D}}=F_{Y \mathrm{D}}=F_{\mathrm{ZD}}=1 / 3$ et $\cos ^{2} \alpha_{X \mathrm{D}}=\cos ^{2} \alpha_{Y \mathrm{D}}=$ $\cos ^{2} \alpha_{Z D}=1 / 3$, soit,

$$
\varphi_{c}=\varphi_{M}\left\{\frac{2 \varphi_{M}+\varphi_{D}-2 c_{D}\left(\varphi_{M}-\varphi_{D}\right)}{2 \varphi_{M}+\varphi_{D}+c_{D}\left(\varphi_{M}-\varphi_{D}\right)}\right\}
$$

qui est l'une des diverses expressions de l'équation de Maxwell-Clausius-Mossoti.

Comme l'équation de Maxwell qu'elle généralise, l'équation (9) n'est valable que pour les milieux à faible concentration, c'est-à-dire sans interaction entre particules.

1.1 CAS D'Une CONCENTRATION Plus FORTE. On considère maintenant un milieu biphasé représenté par l'équation générale (9), comme un milieu homogène de propriété $\varphi_{\mathrm{c}}$ et l'on $\mathrm{y}$ introduit à nouveau une faible quantité de particules, avec une concentration $\mathrm{d} c_{\mathrm{D}}$. Alors $\varphi_{\mathrm{c}}$ devient $\varphi_{\mathrm{c}}+\mathrm{d} \varphi_{\mathrm{c}}$; $\varphi_{M}$ devient $\varphi_{c} ; c_{M}+c_{D}+\mathrm{d} c_{D}=1$ et l'équation (9) devient, après division du numérateur et du dénominateur par $1-c_{\mathrm{D}}-\mathrm{d} c_{\mathrm{D}}$ :

$$
\varphi_{\mathrm{c}}+\mathrm{d} \varphi_{\mathrm{c}} \sim \varphi_{\mathrm{c}}\left\{\frac{1+\left[\frac{\mathrm{d} c_{\mathrm{D}}}{1-c_{\mathrm{D}}-\mathrm{d} c_{\mathrm{D}}}\right] \varphi_{\mathrm{D}} \Omega}{1+\left[\frac{\mathrm{d} c_{\mathrm{D}}}{1-c_{\mathrm{D}}-\mathrm{d} c_{\mathrm{D}}}\right] \varphi_{\mathrm{c}} \Omega}\right\}
$$

d'où l'on tire :

$$
\frac{d c_{D}}{1-c_{D}-d c_{D}}=\frac{d \varphi_{c}}{\varphi_{c}\left(\varphi_{D}-\varphi_{c}-d \varphi_{c}\right) \Omega} .
$$

Avec les relations de simplification, $\mathrm{d} c_{\mathrm{D}} \ll 1-c_{\mathrm{D}}$ et $\mathrm{d} \varphi_{\mathrm{c}} \ll \varphi_{\mathrm{c}}$, on obtient l'équation approchée :

$$
\frac{\mathrm{d} c_{\mathrm{D}}}{1-c_{\mathrm{D}}}=\frac{\mathrm{d} \varphi_{\mathrm{c}}}{\varphi_{\mathrm{c}}\left(\varphi_{\mathrm{D}}-\varphi_{\mathrm{c}}\right)\left[\frac{\cos ^{2} \alpha_{X \mathrm{D}}}{\varphi_{\mathrm{c}}+\left(\varphi_{\mathrm{D}}-\varphi_{\mathrm{c}}\right) F_{X \mathrm{D}}}+\frac{\cos ^{2} \alpha_{Y \mathrm{D}}}{\varphi_{\mathrm{c}}+\left(\varphi_{\mathrm{D}}-\varphi_{\mathrm{c}}\right) F_{Y \mathrm{D}}}+\frac{\cos ^{2} \alpha_{Z \mathrm{D}}}{\varphi_{\mathrm{c}}+\left(\varphi_{\mathrm{D}}-\varphi_{\mathrm{c}}\right) F_{Z \mathrm{D}}}\right]}
$$


Il s'agit maintenant d'intégrer (14),

- au premier membre, entre les limites de concentration : 0 et $c_{\mathrm{D}}$;

- au second membre, entre les limites des propriétés de champ : $\varphi_{\mathrm{M}}$ et $\varphi_{\mathrm{c}}$.

Divisant par $\varphi_{\mathrm{D}}^{2}$ les numérateur et dénominateur du deuxième membre de l'équation (14) et posant, pour simplifier l'écriture :

$$
\begin{aligned}
\varphi_{\mathrm{c}} / \varphi_{\mathrm{D}}=t ; c_{\mathrm{D}} & =x ; \quad F_{X \mathrm{D}}=\lambda ; \quad F_{Y \mathrm{D}}=\mu ; \\
F_{Z \mathrm{D}} & =\nu, \text { d'où } \lambda+\mu+\nu=1 \\
\cos ^{2} \alpha_{X \mathrm{D}} & =\ell ; \quad \cos ^{2} \alpha_{Y \mathrm{D}}=m ; \\
\cos ^{2} \alpha_{Z \mathrm{D}} & =n, \text { d'où } \ell+m+n=1
\end{aligned}
$$

on obtient :

$$
\frac{\mathrm{d} x}{1-x}=R(t) \mathrm{d} t
$$

avec

$$
R(t)=\frac{1}{\frac{l}{\frac{1}{1-t}+\frac{\lambda}{t}}+\frac{m}{\frac{1}{1-t}+\frac{\mu}{t}}+\frac{n}{\frac{1}{1-t}+\frac{\nu}{t}}}
$$

Après réduction, on trouve :

$$
R(t)=\frac{P(t)}{Q(t)}
$$

avec :

$$
\begin{gathered}
P(t)=\{t(1-\lambda)+\lambda\}\{t(1-\mu)+\mu\} \times \\
\times\{t(1-\nu)+\nu\} \\
Q(t)=t(1-t)\left(u t^{2}+v t+w\right) \\
u=\ell(1-\mu)(1-\nu)+m(1-\nu)(1-\lambda)+ \\
+. n(1-\lambda)(1-\mu) \\
v=\ell(\mu+\nu-2 \mu \nu)+m(\lambda+\nu-2 \lambda \nu) \\
+n(\lambda+\mu-2 \lambda \mu) \\
w=\ell \mu \nu+m \lambda \nu+n \lambda \mu .
\end{gathered}
$$

Dans la suite, on posera :

$$
T(t)=u t^{2}+v t+w=u\left(t-t_{1}\right)\left(t-t_{2}\right)
$$

avec :

$$
\begin{array}{r}
t_{1}=\frac{-v+\sqrt{v^{2}-4 u w}}{2 u} \\
\text { et } t_{2}=\frac{-v-\sqrt{v^{2}-4 u w}}{2 u}
\end{array}
$$

et on écrira $P(t)$ sous la forme :

$$
P(t)=C_{1} t^{3}+C_{2} t^{2}+C_{3} t+q
$$

avec :

$C_{1}=(1-\lambda)(1-\mu)(1-\nu)$

$$
\begin{aligned}
C_{2}= & (1-\lambda)(1-\mu) \nu+(1-\nu)(1-\lambda) \mu+ \\
& +(1-\mu)(1-\nu) \lambda \\
C_{3}= & (1-\lambda) \mu \nu+(1-\mu) \lambda \nu+(1-\nu) \lambda \mu \\
q= & \lambda \mu \nu
\end{aligned}
$$

(*) Lorsque les racines de $Q(t)$ sont toutes distinctes, on peut décomposer $R(t)$ sous la forme :

$$
R(t)=\frac{A}{t}+\frac{B}{1-t}+\frac{C}{t-t_{1}}+\frac{D}{t-t_{2}} .
$$

(**) Lorsqu'elles ne sont pas toutes distinctes, la décomposition (32) n'est plus nécessairement valable et il faut calculer les valeurs correspondantes de $R(t)$. Notons tout d'abord que la racine $t=1$ est toujours simple, car $t_{1}$ et $t_{2}$ sont toujours négatifs ou nuls (cf. annexe 1). Par contre, la racine $t=0$ est multiple si $w=0$. D'après (24), ceci n'est possible que si l'on a :

- soit $\lambda=0$ et $\ell=0$, c'est-à-dire si les inclusions sont des cylindres $(\lambda=0 \Leftrightarrow a=\infty)$ d'axes perpendiculaires au champ $\left(\ell=0 \Leftrightarrow \alpha_{X \mathrm{D}}=\pi / 2\right)$. Utilisant les équations (20) à (24), on obtient :

$$
\begin{aligned}
& R(t)= \\
& =\frac{1}{t(1-t)} \frac{\{t(1-\mu)+\mu\}\{t \mu+(1-\mu)\}}{(1-t)\{m(1-2 \mu)+\mu\}+t}
\end{aligned}
$$

et (32) reste valable avec $C=0$.

- soit $\lambda=\mu=0$, c'est-à-dire si les inclusions sont des feuillets. La direction du champ peut alors être quelconque. D'après (18), on a :

$$
R(t)=\frac{1}{(1-t)\{n t+(1-n)\}}
$$

et (32) reste encore valable avec $A=C=0$.

Enfin, on peut avoir $t_{1}=t_{2}=-1$ pour $\ell=1$, $\mu=\nu=1 / 2$, c'est-à-dire si les inclusions sont des cylindres de révolution, d'axes parallèles au champ, on a alors :

$$
R(t)=\frac{1}{1-t}
$$

et (32) reste toujours valable avec $A=C=D=0$.

1.2 Calcul DES COEFFICIENTS DE L'ÉQUATION (32). - Poursuivant la décomposition en éléments simples, il vient :

$$
A=\frac{\lambda \mu \nu}{\ell_{\mu \nu}+m \lambda \nu+n \lambda \mu}=\frac{q}{w}
$$

$$
\begin{aligned}
& A-B+C+D= \frac{(\lambda-1)(\mu-1)(\nu-1)}{\ell(\mu-1)(\nu-1)+m(\lambda-1) \times} \\
& \times(\nu-1)+n(\lambda-1)(\mu-1) \\
&=-\frac{C_{1}}{u}
\end{aligned}
$$

$$
B=1
$$




$$
\begin{aligned}
C & =\frac{P\left(t_{1}\right)}{t_{1}\left(1-t_{1}\right) u\left(t_{1}-t_{2}\right)} \\
& =\frac{P\left(t_{1}\right)}{u\left(1-t_{1}\right)\left(t_{1}^{2}-\frac{w}{u}\right)}=\frac{P\left(t_{1}\right)}{Q_{1}\left(t_{1}\right)} \\
D & =\frac{P\left(t_{2}\right)}{t_{2}\left(1-t_{2}\right) u\left(t_{2}-t_{1}\right)} \\
& =\frac{P\left(t_{2}\right)}{u\left(1-t_{2}\right)\left(t_{2}^{2}-\frac{w}{u}\right)}=\frac{P\left(t_{2}\right)}{Q_{1}\left(t_{2}\right)}
\end{aligned}
$$

en posant dans (37) et (38),

$$
\begin{aligned}
Q_{1}(t) & =(1-t)\left(u t^{2}-w\right) \\
& =\left(u t^{2}+v t+w\right)\left(a^{\prime} t+b^{\prime}\right)+(a t+b) .
\end{aligned}
$$

Après avoir identifié les deux membres de (39), on trouve finalement :

$$
\begin{aligned}
& a^{\prime}=-1 ; \quad b^{\prime}=1+(v / u) ; \\
& a=2 w-v\{1+(v / u)\}
\end{aligned}
$$

et $b=w\{-1-(1+v / u)\}=-w(2+v / u)(40)$

de même,

$$
P(t)=\left(u t^{2}+v t+w\right)\left(c^{\prime} t+d^{\prime}\right)+(c t+d)
$$

Après avoir identifié les deux membres de (41), on trouve, avec la notation utilisée dans les équations (27) à (31) :

$$
\begin{aligned}
c^{\prime} & =C_{1} / u ; d^{\prime}=\left\{C_{2}-\left(v C_{1} / u\right)\right\} / u ; \\
c & =C_{3}-\left(w C_{1} / u\right)-v\left\{C_{2}-\left(v C_{1} / u\right)\right\} / u \\
\text { et } \quad d & =q-w\left\{C_{2}-\left(v C_{1} / u\right)\right\} / u
\end{aligned}
$$

Soit, en utilisant (37), (38), (39) et (41) :

$$
P\left(t_{i}\right)=c t_{i}+d ; \quad Q_{1}\left(t_{i}\right)=a t_{i}+b ;
$$

avec

$$
i=1,2 \text {, }
$$

et donc,

$$
C=R_{1}\left(t_{1}\right) \text {, et } D=R_{1}\left(t_{2}\right),
$$

avec :

$$
R_{1}(t)=\frac{c t+d}{a t+b}
$$

$N . B$. On trouvera en annexe 1 , les domaines de variation de $u, v, w$, des racines $t_{1}$ et $t_{2}$ et de $(A-B+C+D)$.

Les coefficients $C$ et $D$ sont ainsi exprimés en fonction de sept variables $u, v, w, q, C_{1}, C_{2}$, et $C_{3}$. Ils ne dépendent en fait que de quatre variables indépendantes $\ell, m, \lambda$, et $\mu$ par exemple. Si l'on utilise les premières, on devra manipuler des polynômes de degré 9 , degré de $C_{3} u$ qui apparaît dans l'expression de $c$ (Eq. (42)), après réduction au même dénominateur. On peut réduire à trois le degré de ces polynômes et aussi le nombre de leurs termes, en utilisant les quatre variables suivantes : $q$ (Eq. (31)), $w$ (Eq. (24)),

et

$$
\begin{aligned}
& r=\lambda \mu+\mu \nu+\lambda \nu \\
& s=\ell \lambda+m \mu+n \nu .
\end{aligned}
$$

D'où, d'après (29) et en utilisant (16), puis (15),

$$
C_{2}=\lambda^{2}+\mu^{2}+\nu^{2}+3 q=1-2 r+3 q \text {. }
$$

De même, d'après (28), puis (30) et utilisant (15) dans les deux cas,

$$
\text { et } \quad \begin{aligned}
& C_{1}=r-q \\
& C_{3}=r-3 q
\end{aligned}
$$

(22) devient alors :

$$
u=s+w
$$

(23) devient alors :

$$
v=1-u-w=1-s-2 w
$$

(26) devient alors :

$$
\begin{aligned}
t_{1} & =\frac{s+2 w-1+\sqrt{(1-s)^{2}-4 w}}{2(s+w)} \\
\text { et } \quad t_{2} & =\frac{s+2 w-1-\sqrt{(1-s)^{2}-4 w}}{2(s+w)}
\end{aligned}
$$

Les coefficients $a, b, c$ et $d$ valent respectivement :

$$
\text { et } \quad \begin{aligned}
a= & (-1+s+3 w+s w) /(s+w) \\
b= & -w(1+s) /(s+w) \\
& s^{2}(1-q)+s(-w r-q+3 w-1)+ \\
c & =\frac{+w(2 w+q-2 r-1)+r-q}{(s+w)^{2}}
\end{aligned}
$$

et

$$
d=\left\{s^{2} q+s w(r-1)+w(r-q-w)\right\} /(s+w)^{2} .
$$

1.3 CAS DE L'ELLIPSOÏDE DE RÉVOLUTION : DÉCOMPOSITION DE $R(t)$. - Lorsque les inclusions ont en moyenne la forme d'un ellipsoïde de révolution (hypothèse d'Ondracek), on a la relation $\lambda=\mu$ et donc, $\nu=1-2 \lambda$. Portant ces valeurs dans l'équation (18), et tenant compte de (16), on obtient :

$$
R(t)=\frac{\{t(1-\lambda)+\lambda\}\{2 \lambda t+(1-2 \lambda)\}}{t(1-t)\{t(n-3 n \lambda+2 \lambda)+(1-n-2 \lambda+3 n \lambda)\}}
$$


On voit que $R(t)$ ne dépend que de deux paramètres $n$ et $\lambda$. Les paramètres $\ell$ et $m$ peuvent être choisis arbitrairement, pourvu qu'on ait $\ell \geqslant 0, m \geqslant 0$ et $\ell+m+n=1$.

Revenant aux notations d'Ondracek, en remplaçant $t$ par $\varphi_{\mathrm{c}} / \varphi_{\mathrm{D}}, \lambda$ par $F_{\mathrm{D}}$ et $n$ par $\cos ^{2} \alpha_{\mathrm{D}}$, on obtient :

$$
R(t)=\frac{\left[\varphi_{\mathrm{c}}\left(1-F_{\mathrm{D}}\right)+\varphi_{\mathrm{D}} F_{\mathrm{D}}\right]\left[2 F_{\mathrm{D}} \varphi_{\mathrm{c}}+\left(1-2 F_{\mathrm{D}}\right) \varphi_{\mathrm{D}}\right]}{\varphi_{\mathrm{c}}\left(\varphi_{\mathrm{D}}-\varphi_{\mathrm{c}}\right)\left\{\frac{\varphi_{\mathrm{c}}}{\varphi_{\mathrm{D}}}\left(\cos ^{2} \alpha_{\mathrm{D}}-3 F_{\mathrm{D}} \cos ^{2} \alpha_{\mathrm{D}}+2 F_{\mathrm{D}}\right)+\left(1-\cos ^{2} \alpha_{\mathrm{D}}-2 F_{\mathrm{D}}+3 F_{\mathrm{D}} \cos ^{2} \alpha_{\mathrm{D}}\right)\right\}}
$$

et puisque $\frac{\mathrm{d} c_{\mathrm{D}}}{1-c_{\mathrm{D}}}=R(t) \mathrm{d} t=R(t) \mathrm{d} \varphi_{\mathrm{c}} / \varphi_{\mathrm{D}}$, alors :

$$
\left.\frac{\mathrm{d} c_{\mathrm{D}}}{1-c_{\mathrm{D}}}=\mathrm{d} \varphi_{\mathrm{c}} \frac{2 F_{\mathrm{D}}\left(1-F_{\mathrm{D}}\right) \varphi_{\mathrm{c}}^{2}+\left(1-3 F_{\mathrm{D}}+4 F_{\mathrm{D}}^{2}\right) \varphi_{\mathrm{c}} \varphi_{\mathrm{D}}+F_{\mathrm{D}}\left(1-2 F_{\mathrm{D}}\right) \varphi_{\mathrm{D}}^{2}}{\varphi_{\mathrm{c}}\left(\varphi_{\mathrm{D}}-\varphi_{\mathrm{c}}\right)\left\{\varphi_{\mathrm{c}}\left[2 F_{\mathrm{D}}+\cos ^{2} \alpha_{\mathrm{D}}\left(1-3 F_{\mathrm{D}}\right)\right]+\varphi_{\mathrm{D}}\left[1-2 F_{\mathrm{D}}-\cos ^{2} \alpha_{\mathrm{D}}\left(1-3 F_{\mathrm{D}}\right)\right]\right.}\right\}
$$

qui est l'équation (26) d'Ondracek.

La décomposition en éléments simples s’écrit :

$$
R(t)=\frac{A}{t}+\frac{B}{1-t}+\frac{C^{\prime}}{t-t_{1}^{\prime}} .
$$

En posant :

$$
\begin{aligned}
& a_{1}=n-3 n \lambda+2 \lambda \\
& t_{1}^{\prime}=1-\left(1 / a_{1}\right),
\end{aligned}
$$

le dénominateur de (57) vaut alors : $a_{1} t(1-t)\left(t-t_{1}^{\prime}\right)$ et l'on calcule :

$$
\begin{aligned}
& A=\frac{\lambda(1-2 \lambda)}{1-a_{1}} \\
& B=1 \\
& C^{\prime}=1+\lambda \frac{a_{1}+2 \lambda-2}{a_{1}\left(1-a_{1}\right)}
\end{aligned}
$$

soit, en revenant aux notations d'Ondracek :

$$
\begin{aligned}
A= & \frac{F_{\mathrm{D}}\left(1-2 F_{\mathrm{D}}\right)}{1-\cos ^{2} \alpha_{\mathrm{D}}\left(1-3 F_{\mathrm{D}}\right)-2 F_{\mathrm{D}}} ; \quad B=1 \\
C^{\prime}= & 1-\frac{F_{\mathrm{D}}\left(1-2 F_{\mathrm{D}}\right)}{1-2 F_{\mathrm{D}}-\cos ^{2} \alpha_{\mathrm{D}}\left(1-3 F_{\mathrm{D}}\right)}- \\
& -\frac{2 F_{\mathrm{D}}\left(1-F_{\mathrm{D}}\right)}{2 F_{\mathrm{D}}+\cos ^{2} \alpha_{\mathrm{D}}\left(1-3 F_{\mathrm{D}}\right)} .
\end{aligned}
$$

On retrouve bien les coefficients $R$ et $S$ d'Ondracek, avec $R=A, S=-B$. Le coefficient $C^{\prime}$ est le coefficient $T$ d'Ondracek après rectification d'une erreur sur le signe de $T\left(C^{\prime}=-T\right)$.

$N . B$. On trouvera en annexe 2, les domaines de variation de $a_{1}, t_{1}^{\prime}, A$ et $C^{\prime}$ pour $\lambda=\mu$ (cas de l'ellipsoïde de révolution).

1.4 EQUATION GÉNÉRALE (CAS DE L'ELLIPSOİDE QUELCONQUE). - Reprenant les équations (17), (18) et (32), il vient :

$$
\begin{aligned}
\frac{\mathrm{d} x}{1-x} & =\frac{\mathrm{d} c_{\mathrm{D}}}{1-c_{\mathrm{D}}}= \\
& =A \frac{\mathrm{d} t}{t}+B \frac{\mathrm{d} t}{1-t}+C \frac{\mathrm{d} t}{t-t_{1}}+D \frac{\mathrm{d} t}{t-t_{2}}
\end{aligned}
$$

dont la solution est donnée par :

$$
\begin{aligned}
&-\ln \left(1-c_{\mathrm{D}}\right)=A \ln \frac{t}{t_{\mathrm{M}}}-\ln \left|\frac{1-t}{1-t_{\mathrm{M}}}\right|+ \\
&+C \ln \left|\frac{t-t_{1}}{t_{\mathrm{M}}-t_{1}}\right|+D \ln \left|\frac{t-t_{2}}{t_{\mathrm{M}}-t_{2}}\right| .
\end{aligned}
$$

Comme on a montré que $t_{1}$ et $t_{2}$ sont toujours négatifs (cf. annexe 1), on peut enlever les deux derniers signes de valeur absolue dans (64), et réécrire cette équation sous la forme :

$$
F(t)=\frac{F\left(t_{n}\right)}{1-c_{\mathrm{D}}}
$$

avec :

$$
F(t)=\frac{t^{A}\left(t+\left|t_{1}\right|\right)^{C}\left(t+\left|t_{2}\right|\right)^{D}}{|1-t|}
$$

Pour étudier la variation de $F(t)$, on examine le signe de sa dérivée, d'où l'on déduit : $F(t)$ monotone croissante dans l'intervalle

$$
0 \leqslant t \leqslant 1
$$

$F(t)$ monotone décroissante dans l'intervalle

$$
1 \leqslant t<+\infty \text {. }
$$

L'équation (63) admet donc une solution et une seule dans chacun des intervalles $0 \leqslant t \leqslant 1$ et $1 \leqslant t<+\infty$.

Aux bornes, on a, d'après (66),

$$
F(t)_{t \rightarrow 0} \sim\left|t_{1}\right|^{C}\left|t_{2}\right|^{D} t^{A} .
$$

D'après (34),

$$
A>0 \text { et donc } F(0)=0 .
$$


Pour $t \rightarrow 1$,

$F(t)_{t \rightarrow 1} \sim\left(1+\left|t_{1}\right|\right)^{C}\left(1+\left|t_{2}\right|\right)^{D} \frac{1}{|1-t|}$.

Enfin, pour $t \rightarrow \infty$,

$$
F(t)_{t \rightarrow \infty} \sim t^{(A+C+D-1)}
$$

et, comme d'après (35) et (36), l'exposant de $t$ est négatif,

$$
\lim _{t \rightarrow \infty} F(t)=0
$$

Cas général.

Il résulte de (65) et (66) :

$$
\begin{aligned}
1-c_{\mathrm{D}}=\left(\frac{t_{\mathrm{n}}}{t}\right)^{A} & \left|\frac{1-t}{1-t_{\mathrm{n}}}\right| \times \\
& \times\left(\frac{t_{\mathrm{n}}-t_{1}}{t-t_{1}}\right)^{C}\left(\frac{t_{\mathrm{n}}-t_{2}}{t-t_{2}}\right)^{D}
\end{aligned}
$$

soit, en remplaçant $t$ par $\varphi_{\mathrm{c}} / \varphi_{\mathrm{D}}$ et $t_{\mathrm{n}} \operatorname{par} \varphi_{\mathrm{M}} / \varphi_{\mathrm{D}}$,

$$
\begin{aligned}
1-c_{\mathrm{D}} & =\left(\frac{\varphi_{\mathrm{M}}}{\varphi_{\mathrm{c}}}\right)^{A}\left|\frac{\varphi_{\mathrm{D}}-\varphi_{\mathrm{c}}}{\varphi_{\mathrm{D}}-\varphi_{\mathrm{M}}}\right| \times \\
& \times\left(\frac{\varphi_{\mathrm{M}}-\varphi_{\mathrm{D}} t_{1}}{\varphi_{\mathrm{c}}-\varphi_{\mathrm{D}} t_{1}}\right)^{C}\left(\frac{\varphi_{\mathrm{M}}-\varphi_{\mathrm{D}} t_{2}}{\varphi_{\mathrm{c}}-\varphi_{\mathrm{D}} t_{2}}\right)^{D} .
\end{aligned}
$$

Dans ce résultat, les cinq constantes $A, C, D$, $t_{1}$ et $t_{2}$ s'expriment en fonction de $q, r, s$ et $w$. Exprimant ces constantes avec les notations $F_{i \mathrm{D}}$ et $\cos ^{2} \alpha_{i \mathrm{D}}$, il vient :

$$
\begin{aligned}
q= & F_{X \mathrm{D}} F_{Y \mathrm{D}} F_{Z \mathrm{D}} \\
r= & F_{X \mathrm{D}} F_{Y \mathrm{D}}+F_{Y \mathrm{D}} F_{Z \mathrm{D}}+F_{Z \mathrm{D}} F_{X \mathrm{D}} \\
s= & F_{X \mathrm{D}} \cos ^{2} \alpha_{X \mathrm{D}}+F_{Y \mathrm{D}} \cos ^{2} \alpha_{Y \mathrm{D}}+ \\
& +F_{Z \mathrm{D}} \cos ^{2} \alpha_{Z \mathrm{D}} \\
w= & F_{X \mathrm{D}} F_{Y \mathrm{D}} \cos ^{2} \alpha_{Z \mathrm{D}}+F_{Y \mathrm{D}} F_{Z \mathrm{D}} \cos ^{2} \alpha_{X \mathrm{D}}+ \\
& +F_{Z \mathrm{D}} F_{X \mathrm{D}} \cos ^{2} \alpha_{Y \mathrm{D}}
\end{aligned}
$$

d'où, d'après (34) :

$$
A=\frac{q}{w}=\frac{F_{X \mathrm{D}} F_{Y \mathrm{D}} F_{Z \mathrm{D}}}{F_{X \mathrm{D}} F_{Y \mathrm{D}} \cos ^{2} \alpha_{Z \mathrm{D}}+F_{Y \mathrm{D}} F_{Z \mathrm{D}} \cos ^{2} \alpha_{X \mathrm{D}}+F_{Z \mathrm{D}} F_{X \mathrm{D}} \cos ^{2} \alpha_{Y \mathrm{D}}}
$$

et d'après (37), (38), (39), (41) et (42) :

$C=\frac{\left\{s^{2}(1-q)+s(3 w-w r-q-1)+w(2 w+q-2 r-1)+r-q\right\} t_{1}+s^{2} q+s w(r-1)+w(r-q-w)}{(s+w)\left[(-1+s+3 w+s w) t_{1}-w(1+s)\right]}$

$D=\frac{\left\{s^{2}(1-q)+s(3 w-w r-q-1)+w(2 w+q-2 r-1)+r-q\right\} t_{2}+s^{2} q+s w(r-1)+w(r-q-w)}{(s+w)\left[(-1+s+3 w+s w) t_{2}-w(1+s)\right]}$

avec

$$
t_{1}=\frac{(s+2 w-1)+\sqrt{(1-s)^{2}-4 w}}{2(s+w)}
$$

et

$$
t_{2}=\frac{(s+2 w-1)-\sqrt{(1-s)^{2}-4 w}}{2(s+w)}
$$

Cas particuliers.

- Pour $c_{\mathrm{D}}=0$, le composite se réduit à la matrice, et la solution de (74) est :

$$
t=t_{\mathrm{n}}, \text { soit } \varphi_{\mathrm{c}}=\varphi_{\mathrm{M}} .
$$

- Pour $c_{\mathrm{D}}=1$, le composite se réduit à la phase dispersée, et la solution de (74) est :

$$
t=1, \text { soit } \varphi_{\mathrm{c}}=\varphi_{\mathrm{D}}
$$

Pour une phase dispersée solide, la solution (86) n'est généralement pas atteinte

(+) pour une phase dispersée constituée de cylindres, la densité maximum de cylindres est égale à la densité maximum de cercles dans un plan, c'est-àdire :

$$
c_{\mathrm{D}} \leqslant \frac{\pi}{2 \sqrt{3}} \sim 0,91
$$


$(++)$ pour une phase dispersée constituée de sphères de même rayon en empilement compact, $\lambda=\mu=\nu=1 / 3$ et :

$$
c_{\mathrm{D}} \leqslant \frac{\pi}{3 \sqrt{2}} \sim 0,74
$$

$(+++)$ pour une phase dispersée constituée de feuillets, on peut avoir $c_{D}=1$, soit $\varphi_{c}=\varphi_{D}$. La solution (86) est dans ce cas atteinte.

- Si $t_{\mathrm{n}} \gg 1$, soit $\varphi_{\mathrm{M}} / \varphi_{\mathrm{D}} \gg 1$ et donc, $t=\varphi_{\mathrm{c}} / \varphi_{\mathrm{D}} \gg 1$, alors

$$
\begin{aligned}
\lim _{t \rightarrow \infty} F(t) \sim t^{A-B+C+D} \Rightarrow \\
\quad \Rightarrow t \sim t_{\mathrm{n}}\left(1-c_{\mathrm{D}}\right)^{-1 /(A-B+C+D) .}
\end{aligned}
$$

Ceci généralise l'équation de Pejsa (1980, p. 329) :

$$
\varphi_{c}=\varphi_{M}\left(1-c_{D}\right)^{\left[\frac{\cos ^{2} \alpha_{D}-1}{F_{D}-1}+\frac{\cos ^{2} \alpha_{D}}{2 F_{D}}\right]}
$$

équation approchée pour le cas de l'ellipsoïde de révolution et d'un fort contraste de conductivité entre matrice et phase dispersée.

En effet, dans le cas de l'ellipsoïde de révolution, $\lambda=\mu$ et donc $\nu=1-2 \lambda$. Reprenant (35) et (36), et en tenant compte de (16), on retrouve bien l'exposant de l'équation de Pejsa. alors :

- Si $t_{\mathrm{n}} \ll\left|t_{1}\right|$ et donc $t \ll\left|t_{1}\right|$, (65) devient

$$
\begin{gathered}
\left|t_{1}\right|^{C}\left|t_{2}\right|^{D} t^{A} \sim \frac{\left|t_{1}\right|^{C}\left|t_{2}\right|^{D} t_{n}^{A}}{1-c_{\mathrm{D}}} \Rightarrow \\
\Rightarrow t \sim t_{\mathrm{n}}\left(1-c_{\mathrm{D}}\right)^{-1 / A} .
\end{gathered}
$$

\section{Prise en compte des données de la microscopie.}

En pratique, on peut disposer de deux types d'échantillons, parallélépipédiques et cylindriques. On ne traite ici que du cas du bloc parallélépipédique ou cubique.

On admet que son étude stéréologique, suivant des coupes mutuellement perpendiculaires $A, B, C$ (et éventuellement suivant d'autres coupes) a révélé l'existence d'un ellipsoïde moyen équivalent (E.M.E.), représentatif de la forme des pores, ou de celles des grains de l'une des phases en présence sur l'ensemble du volume, et l'existence d'une ellipse moyenne équivalente (e.m.e.) associée à chacune des coupes $A, B, C$. Sous réserve que l'échantillon soit suffisamment homogène (au sens usuel de ce terme), on peut admettre que les trois e.m.e. en question sont elles-mêmes des coupes diamétrales de l'E.M.E.
La définition de la forme moyenne équivalente, selon Riss et Grolier (1986) et Riss (1987), et le principe de sa mise en œuvre sont exposés maintenant, à l'intention du praticien. Une forme moyenne équivalente (F.M.E.) à un ensemble de particules d'un agrégat est définie par deux données conjointes : une forme canonique et une taille telle que les fonctionnelles $V, S, \bar{H}$ (volume, aire de la surface et hauteur moyenne projetée) de la F.M.E. soient égales aux moyennes des fonctionnelles des particules. En particulier, nous appellerons (s'il existe) ellipsoïde moyen équivalent (E.M.E.) à l'ensemble des grains de l'une des phases constitutives de la roche étudiée, un ellipsoïde triaxial défini par sa forme (rapports des demi-axes), par sa taille et dont les fonctionnelles $V, S, \bar{H}$ sont égales aux moyennes $\bar{V}, \bar{S}, \bar{H}$ de ces mêmes fonctionnelles estimées sur la population de grains étudiés. Les méthodes stéréologiques à mettre en œuvre dans cette estimation sont maintenant bien connues.

Des équations stéréologiques suivantes (où le signe double barre désigne la moyenne d'un paramètre qui est lui-même une moyenne) :

$\left.\begin{array}{l}N_{\mathrm{A}}=N_{\mathrm{V}} \overline{\bar{H}}, \quad \overline{\bar{B}}=\pi \bar{S} /(4 \overline{\bar{H}}), \quad \overline{\bar{A}}=\bar{V} / \overline{\bar{H}} \\ N_{\mathrm{L}}=N_{\mathrm{V}} \bar{S} / 4, \overline{\bar{L}}=4 \bar{V} / \bar{S} \text { et } \overline{\overline{L^{4}}}=12 \overline{V^{2}} /(\pi \bar{S})\end{array}\right\}$

et où, $\overline{\bar{H}}$ (qui est égal à $\bar{H}$ ) est la moyenne de la hauteur moyenne projetée des particules et de la F.M.E.,

$\bar{S}$ (qui est égal à $S$ ) l'aire moyenne de la surface des particules,

$\bar{V}$ (qui est égal à $V$ ) le volume moyen des particules,

$N_{\mathrm{V}}$ le nombre de particules par unité de volume,

$N_{\mathrm{A}}$ le nombre de particules par unité de surface de la préparation,

$\overline{\bar{B}}$ la moyenne des périmètres des coupes planes des particules,

$\overline{\bar{A}}$ l'aire moyenne des coupes planes des particules,

$N_{\mathrm{L}}$ le nombre des particules par unité de longueur des traversées,

$\underline{\bar{L}}$ la moyenne des intercepts linéaires,

$\overline{L^{4}}$ la moyenne de la puissance 4 des intercepts linéaires, on tire les rapports $\bar{V} / \bar{H}, \bar{V} / \bar{S}, \bar{S} / \bar{H}$, $\overline{V^{2}} / \bar{V}$ et, les particules équivalentes ayant toutes le même volume en raison même de la définition de l'E.M.E., on obtient immédiatement les trois fonctionnelles $\bar{H}, S$ et $V$.

On notera que la procédure peut être simplifiée si l'on utilise l'estimateur (non biaisé et pondéré en volume) du volume moyen des particules établi par Jensen \& Gundersen (1985) :

$$
V=(\pi / 3) \overline{L^{3}} \text {. }
$$


Il suffit alors de connaître les deux rapports $V / \bar{H}$ et $S / \bar{H}$ pour obtenir $S$ et $\bar{H}$. On a $E(A)=V / \bar{H}$ et $E(B)=\pi S / 4 \bar{H}$ où $A$ et $B$ sont respectivement l'aire de la surface et le périmètre des particules telles qu'elles apparaissent sur des sections au hasard («I.U.R. sections», Weibel 1981).

En définitive, on détermine l'E.M.E. en se référant à des tables ou à des abaques dressées une fois pour toutes et donnant directement les rapports $a / \bar{H}, b / \bar{H}, c / \bar{H}$ de l'ellipsoïde de fonctionnelles $V$, $S, \bar{H}$ (Riss et Grolier 1986 et Riss 1987). On suppose dans ce qui suit qu'il existe effectivement une E.M.E. (on conçoit en effet qu'il puisse ne pas en être ainsi et que la F.M.E. soit par exemple un polyèdre).

C'est par une méthode analogue (dont les modalités pratiques restent toutefois à expliciter) que l'on pourra déterminer les e.m.e. associées aux coupes $A, B, C$. Toutefois les difficultés de mise en œuvre étant supposées résolues, nous considérons maintenant que l'E.M.E. est connu (par ses demi-axes $a, b$, $c)$; et les e.m.e., par leurs demi-axes $\left(a^{\prime}, b^{\prime}\right)$, $\left(a^{\prime \prime}, b^{\prime \prime}\right),\left(a^{\prime \prime \prime}, b^{\prime \prime \prime}\right)$. Il faut en déduire aussi, afin d'utiliser les formules (75) à (84), l'orientation de l'E.M.E., généralement oblique par rapport au champ appliqué. Autrement dit, il s'agit de trouver les cosinus directeurs de $A_{1}, A_{2}, A_{3}$ par rapport à $\mathrm{O} X_{3}$ (Fig. 1). On traite ici le cas le plus général champ triaxial - et l'on détermine l'orientation de l'E.M.E. par rapport à chacun des axes.

Dans le système d'axes $A_{1}, A_{2}, A_{3}$, l'équation de l'ellipsoïde est :

$$
\frac{A_{1}^{2}}{a^{2}}+\frac{A_{2}^{2}}{b^{2}}+\frac{A_{3}^{2}}{c^{2}}=1
$$

Pour écrire cette équation dans le système d'axes $X_{1}, X_{2}, X_{3}$ lié à l'échantillon et au champ, on écrit la matrice de changement d'axes $\left(a_{i j}\right)$ :

\begin{tabular}{c|ccc} 
& $A_{1}$ & $A_{2}$ & $A_{3}$ \\
\hline$X_{1}$ & $\cos \theta_{1}$ & $\cos \theta_{2}$ & $\cos \theta_{3}$ \\
$X_{2}$ & $\cos \gamma_{1}$ & $\cos \gamma_{2}$ & $\cos \gamma_{3}$ \\
$X_{3}$ & $\cos \alpha_{1}$ & $\cos \alpha_{2}$ & $\cos \alpha_{3}$
\end{tabular}

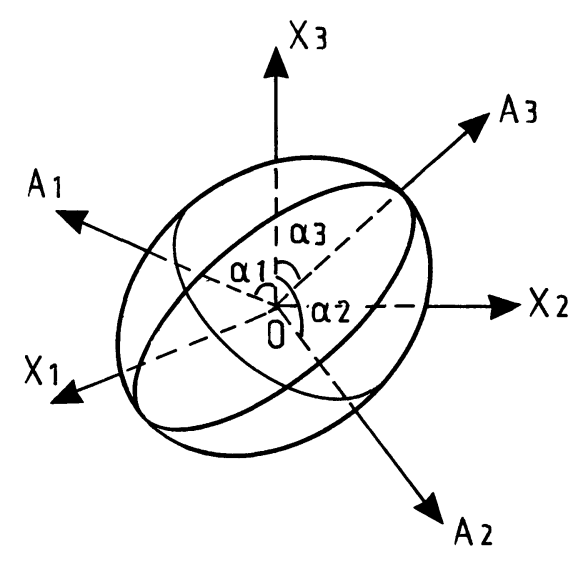

Fig. 1. - Orientation mutuelle des axes principaux de l'ellipsoïde moyen équivalent et d'un champ triaxial $\mathrm{O} X_{1}, \mathrm{O} X_{2}, \mathrm{O} X_{3}$.

[Mutual orientation of the main axes of the average equivalent ellipsoid and of $\mathrm{O} X_{1}, \mathrm{OX}_{2}, \mathrm{O} X_{3}$ triaxial field.]

où

$\theta_{1}, \theta_{2}, \theta_{3}$ sont les angles que fait $X_{1}$ avec $A_{1}$, $A_{2}, A_{3}$;

$\gamma_{1}, \gamma_{2}, \gamma_{3}$ sont les angles que fait $X_{2}$ avec $A_{1}$, $A_{2}, A_{3}$

$\alpha_{1}, \alpha_{2}, \alpha_{3}$ sont les angles que fait $X_{3}$ avec $A_{1}$, $A_{2}, A_{3}$;

Il n'est pas nécessaire de déterminer les neuf valeurs de ces angles ; en effet, du fait des propriétés des matrices orthogonales,

$$
a_{i k} a_{j k}=\delta_{i j} \text { et } a_{k i} a_{k j}=\delta_{i j},
$$

seules trois de ces neuf valeurs sont indépendantes et la matrice est entièrement connue si l'on a déterminé $\alpha_{1}, \alpha_{2}$ et $\gamma_{1}$ par exemple.

2.1 EQUATION DE L'ELLIPSOÏDE DANS LE SYSTÈME D'AXES $X_{1}, X_{2}, X_{3}$. - Portons dans (94) les valeurs :

$$
\begin{aligned}
& A_{1}=X_{1} \cos \theta_{1}+X_{2} \cos \gamma_{1}+X_{3} \cos \alpha_{1} \\
& A_{2}=X_{1} \cos \theta_{2}+X_{2} \cos \gamma_{2}+X_{3} \cos \alpha_{2} \\
& A_{3}=X_{1} \cos \theta_{3}+X_{2} \cos \gamma_{3}+X_{3} \cos \alpha_{3}
\end{aligned}
$$

L'équation de l'ellipsoïde devient alors :

$$
\begin{aligned}
& X_{1}^{2}\left[\frac{\cos ^{2} \theta_{1}}{a^{2}}+\frac{\cos ^{2} \theta_{2}}{b^{2}}+\frac{\cos ^{2} \theta_{3}}{c^{2}}\right]+2 X_{2} X_{3}\left[\frac{\cos \gamma_{1} \cos \alpha_{1}}{a^{2}}+\frac{\cos \gamma_{2} \cos \alpha_{2}}{b^{2}}+\frac{\cos \gamma_{3} \cos \alpha_{3}}{c^{2}}\right] \\
& +X_{2}^{2}\left[\frac{\cos ^{2} \gamma_{1}}{a^{2}}+\frac{\cos ^{2} \gamma_{2}}{b^{2}}+\frac{\cos ^{2} \gamma_{3}}{c^{2}}\right]+2 X_{3} X_{1}\left[\frac{\cos \alpha_{1} \cos \theta_{1}}{a^{2}}+\frac{\cos \alpha_{2} \cos \theta_{2}}{b^{2}}+\frac{\cos \alpha_{3} \cos \theta_{3}}{c^{2}}\right] \\
& +X_{3}^{2}\left[\frac{\cos ^{2} \alpha_{1}}{a^{2}}+\frac{\cos ^{2} \alpha_{2}}{b^{2}}+\frac{\cos ^{2} \alpha_{3}}{c^{2}}\right]+2 X_{1} X_{2}\left[\frac{\cos \theta_{1} \cos \gamma_{1}}{a^{2}}+\frac{\cos \theta_{2} \cos \gamma_{2}}{b^{2}}+\frac{\cos \theta_{3} \cos \gamma_{3}}{c^{2}}\right]=1
\end{aligned}
$$




\subsection{UTILISATION DES COUPES DIAMÉTRALES.}

- Dans le plan perpendiculaire à $\mathrm{O} X_{1}$.

$a^{\prime}, b^{\prime}, \beta^{\prime}$ sont connus. L'équation de l'ellipse

(Fig. 2), rapportée à ses axes principaux est :

$$
\frac{X^{\prime 2}}{a^{\prime 2}}+\frac{Y^{\prime 2}}{b^{\prime 2}}=1
$$

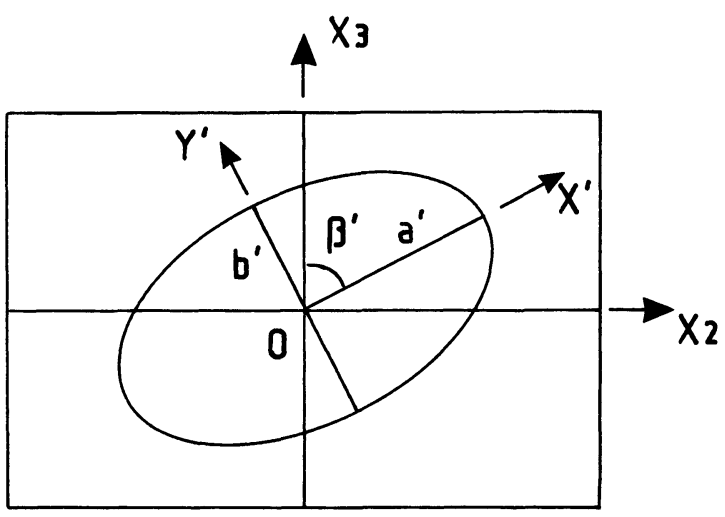

Fig. 2. - Coupe de l'ellipsoïde moyen équivalent par le plan $X_{2} \mathrm{O} X_{3}$.

[The average equivalent ellipsoid cut by the $X_{2} \mathrm{O} X_{3}$ plane.]

La matrice de changement d'axes est :

\begin{tabular}{c|cc} 
& \multicolumn{1}{|c}{$X_{2}$} & $X_{3}$ \\
\hline$X^{\prime}$ & $\sin \beta^{\prime}$ & $\cos \beta^{\prime}$ \\
$Y^{\prime}$ & $-\cos \beta^{\prime}$ & $\sin \beta^{\prime}$
\end{tabular}

soit :

$$
X^{\prime}=X_{2} \sin \beta^{\prime}+X_{3} \cos \beta^{\prime}
$$

et

$$
Y^{\prime}=-X_{2} \cos \beta^{\prime}+X_{3} \sin \beta^{\prime}
$$

Portant ces valeurs dans (97), on obtient :

$$
\begin{aligned}
\left(\frac{X_{2} \sin \beta^{\prime}+X_{3} \cos \beta^{\prime}}{a^{\prime}}\right)^{2}+ \\
\quad+\left(\frac{-X_{2} \cos \beta^{\prime}+X_{3} \sin \beta^{\prime}}{b^{\prime}}\right)_{2}=1
\end{aligned}
$$

c'est-à-dire l'équation de l'ellipse dans le système d'axes $\mathrm{O}_{2} X_{3}$.

$$
\begin{aligned}
X_{2}^{2}\left[\frac{\sin ^{2} \beta^{\prime}}{a^{\prime 2}}\right. & \left.+\frac{\cos ^{2} \beta^{\prime}}{b^{\prime 2}}\right]+X_{3}^{2}\left[\frac{\cos ^{2} \beta^{\prime}}{a^{\prime 2}}+\frac{\sin ^{2} \beta^{\prime}}{b^{\prime 2}}\right]+ \\
& +X_{2} X_{3}\left[\frac{b^{\prime 2}-a^{\prime 2}}{a^{\prime 2} b^{\prime 2}}\right] \sin 2 \beta^{\prime}=1
\end{aligned}
$$

- Dans le plan perpendiculaire à $O X_{2}$. $a^{\prime \prime}, b^{\prime \prime}, \beta^{\prime \prime}$ sont connus. L'équation de l'ellipse (Fig. 3), rapportée à ses axes principaux, est :

$$
\frac{X^{\prime \prime 2}}{a^{\prime \prime 2}}+\frac{Y^{\prime \prime 2}}{b^{\prime \prime 2}}=1
$$

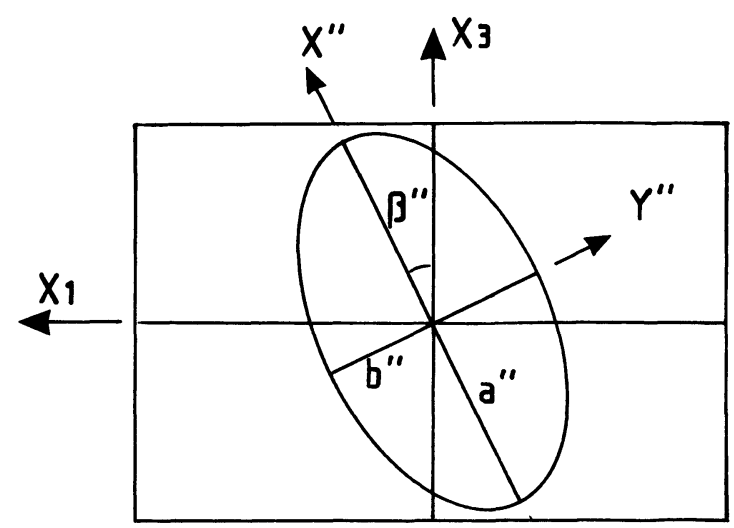

Fig. 3. - Coupe de l'ellipsoïde moyen équivalent par le plan $X_{1} O X_{3}$.

[The average equivalent ellipsoid cut by the $X_{1} \mathrm{O} X_{3}$ plane.]

Procédant de la même manière que pour la coupe précédente, on obtient l'équation de l'ellipse dans le système d'axes $O X_{1} X_{3}$, soit :

$$
\begin{gathered}
X_{1}^{2}\left[\frac{\sin ^{2} \beta^{\prime \prime}}{a^{\prime \prime 2}}+\frac{\cos ^{2} \beta^{\prime \prime}}{b^{\prime \prime 2}}\right]+X_{3}^{2}\left[\frac{\cos ^{2} \beta^{\prime \prime}}{a^{\prime \prime 2}}+\frac{\sin ^{2} \beta^{\prime \prime}}{b^{\prime \prime 2}}\right]+ \\
+X_{1} X_{3}\left[\frac{b^{\prime \prime 2}-a^{\prime \prime 2}}{a^{\prime \prime 2} b^{\prime \prime 2}}\right] \sin 2 \beta^{\prime \prime}=1 . \quad(100)
\end{gathered}
$$

- Dans le plan perpendiculaire à $O X_{3}$. $a^{\prime \prime \prime}, b^{\prime \prime \prime}, \beta^{\prime \prime \prime}$ sont connus. L'équation de l'ellipse (Fig. 4), rapportée à ses axes principaux est :

$$
\frac{X^{\prime \prime 2}}{a^{\prime \prime \prime 2}}+\frac{Y^{\prime \prime \prime 2}}{b^{\prime \prime \prime 2}}=1 \text {. }
$$

Procédant encore de même que pour les coupes précédentes, on obtient :

$$
\begin{aligned}
& X_{1}^{2}\left[\frac{\sin ^{2} \beta^{\prime \prime \prime}}{a^{\prime \prime \prime 2}}+\frac{\cos ^{2} \beta^{\prime \prime \prime}}{b^{\prime \prime \prime 2}}\right]+ \\
& \quad+X_{2}^{2}\left[\frac{\cos ^{2} \beta^{\prime \prime \prime}}{a^{\prime \prime \prime 2}}+\frac{\sin ^{2} \beta^{\prime \prime \prime}}{b^{\prime \prime \prime 2}}\right]+ \\
& \quad+X_{1} X_{2}\left[\frac{b^{\prime \prime \prime 2}-a^{\prime \prime \prime 2}}{a^{\prime \prime \prime 2} b^{\prime \prime \prime 2}}\right] \sin 2 \beta^{\prime \prime \prime}=1 .
\end{aligned}
$$

Dans ce qui suit, on s'inspire en partie de Ramsay (1967, pp. 142-147) qui traite de ce problème géométrique, à propos des relations entre ellipses coupes et ellipsoïdes de déformation. Comparant les équations 


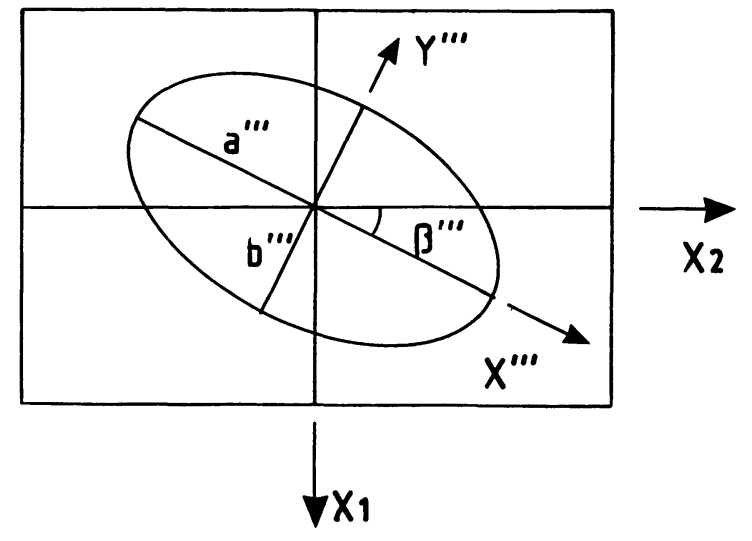

Fig. 4. - Coupe de l'ellipsoïde moyen équivalent par le plan $X_{1} \mathrm{O} X_{2}$.

[The average quivalent ellipsoid cut by the $X_{1} \mathrm{O} X_{2}$ plane.]

des ellipses coupes et les équations déduites de l'équation générale de l'ellipsoïde pour $X_{1}=0$, $X_{2}=0$, et $X_{3}=0$, on trouve les coefficients $A, B, \ldots F$, de l'ellipsoïde oblique. Son équation est de la forme :

$$
\begin{aligned}
A X_{1}^{2}+B X_{2}^{2}+C & X_{3}^{2}+2 D X_{1} X_{2}+ \\
& +2 E X_{2} X_{3}+2 F X_{3} X_{1}=1
\end{aligned}
$$

avec :

$$
\begin{aligned}
A & =\frac{\sin ^{2} \beta^{\prime \prime}}{a^{\prime \prime 2}}+\frac{\cos ^{2} \beta^{\prime \prime}}{b^{\prime \prime 2}} \\
& =\frac{\sin ^{2} \beta^{\prime \prime \prime}}{a^{\prime \prime \prime 2}}+\frac{\cos ^{2} \beta^{\prime \prime \prime}}{b^{\prime \prime \prime 2}} ; \\
B & =\frac{\sin ^{2} \beta^{\prime}}{a^{\prime 2}}+\frac{\cos ^{2} \beta^{\prime}}{b^{\prime 2}} \\
& =\frac{\cos ^{2} \beta^{\prime \prime \prime}}{a^{\prime \prime \prime 2}}+\frac{\sin ^{2} \beta^{\prime \prime \prime}}{b^{\prime \prime \prime 2}} ; \\
C & =\frac{\cos ^{2} \beta^{\prime}}{a^{\prime 2}}+\frac{\sin ^{2} \beta^{\prime}}{b^{\prime 2}} \\
& =\frac{\cos ^{2} \beta^{\prime \prime}}{a^{\prime \prime 2}}+\frac{\sin ^{2} \beta^{\prime \prime}}{b^{\prime \prime 2}} ; \\
D & =\frac{1}{2}\left[\frac{b^{\prime \prime \prime 2}-a^{\prime \prime \prime 2}}{a^{\prime \prime \prime 2} b^{\prime \prime \prime 2}}\right] \sin 2 \beta^{\prime \prime \prime} ; \\
E & =\frac{1}{2}\left[\frac{b^{\prime 2}-a^{\prime 2}}{a^{\prime 2} b^{\prime 2}}\right] \sin 2 \beta^{\prime} ; \\
F & =\frac{1}{2}\left[\frac{b^{\prime \prime 2}-a^{\prime \prime 2}}{a^{\prime \prime 2} b^{\prime \prime 2}}\right] \sin 2 \beta^{\prime \prime}
\end{aligned}
$$

Par ailleurs, les extrémités des axes principaux de l'ellipsoïde sont sur des sphères de rayon $r=a, b, c$, et d'équation :

$$
X_{1}^{2}+X_{2}^{2}+X_{3}^{2}=r^{2}
$$

Différentiant (104) et (103), on obtient :

$$
X_{1} \mathrm{~d} X_{1}+X_{2} \mathrm{~d} X_{2}+X_{3} \mathrm{~d} X_{3}=0
$$

soit

$$
X_{i} \mathrm{~d} X_{i}=0
$$

et

$$
\begin{array}{r}
\left(A X_{1}+D X_{2}+F X_{3}\right) \mathrm{d} X_{1}+\left(B X_{2}+D X_{1}+E X_{3}\right) \times \\
\quad \times \mathrm{d} X_{2}+\left(C X_{3}+E X_{2}+F X_{1}\right) \mathrm{d} X_{3}=0 .
\end{array}
$$

Multipliant (105) par un facteur $q$ arbitraire (multiplicateur de Lagrange), on a :

$$
q X_{1} \mathrm{~d} X_{1}+q X_{2} \mathrm{~d} X_{2}+q X_{3} \mathrm{~d} X_{3}=0 \text {. }
$$

Comparant (106) et (107) et regroupant les termes en $X_{i}$, on obtient :

$$
\left.\begin{array}{l}
(A-q) X_{1}+D X_{2}+F X_{3}=0 \\
D X_{1}+(B-q) X_{2}+E X_{3}=0 \\
F X_{1}+E X_{2}+(C-q) X_{3}=0
\end{array}\right\}
$$

Multipliant respectivement $\operatorname{par} X_{1}, X_{2}$, et $X_{3}$, puis additionnant, on a :

$$
\begin{array}{r}
A X_{1}^{2}+B X_{2}^{2}+C X_{3}^{2}+2 D X_{1} X_{2}+2 E X_{2} X_{3}+ \\
+2 F X_{3} X_{1}-q\left(X_{1}^{2}+X_{2}^{2}+X_{3}^{2}\right)=0
\end{array}
$$

d'où, d'après (103) et (104) :

$$
1-q r^{2}=0 ; \text { soit } q=1 / r^{2} .
$$

Les équations (108) s'écrivent alors :

$$
\left.\begin{array}{l}
\left(A-1 / r^{2}\right) X_{1}+D X_{2}+F X_{3}=0 \\
D X_{1}+\left(B-1 / r^{2}\right) X_{2}+E X_{3}=0 \\
F X_{1}+E X_{2}+\left(C-1 / r^{2}\right) X_{3}=0
\end{array}\right\} \text {. }
$$

d'où l'on tire :

$$
\frac{X_{2}}{X_{3}}=\frac{\left(A-1 / r^{2}\right) E-D F}{D^{2}-\left(B-1 / r^{2}\right)\left(A-1 / r^{2}\right)}=J
$$

et :

$$
\frac{X_{1}}{X_{3}}=\frac{\left(B-1 / r^{2}\right) F-D E}{D^{2}-\left(B-1 / r^{2}\right)\left(A-1 / r^{2}\right)}=K .
$$

Ainsi, $X_{2}^{2}=J^{2} X_{3}^{2}$ et $X_{1}^{2}=K^{2} X_{3}^{2}$; utilisant alors (104), on obtient :

$$
X_{3}= \pm r /\left(1+J^{2}+K^{2}\right)^{1 / 2} .
$$

Les projections $\left(X_{3}\right)_{a},\left(X_{3}\right)_{b},\left(X_{3}\right)_{c}$ de $a, b$, et $c$ sur $X_{3}$ sont données par (112) pour les valeurs respectives $r=a, r=b$, et $r=c$.

Soit :

$$
\begin{aligned}
& a \cos \alpha_{1}= \pm a /\left(1+J_{a}^{2}+K_{a}^{2}\right)^{1 / 2} \\
& b \cos \alpha_{2}= \pm b /\left(1+J_{b}^{2}+K_{b}^{2}\right)^{1 / 2}
\end{aligned}
$$


ou encore :

$$
\cos \alpha_{1}= \pm 1 /\left(1+J_{a}^{2}+K_{a}^{2}\right)^{1 / 2}
$$

et :

$$
\cos \alpha_{2}= \pm 1 /\left(1+J_{b}^{2}+K_{b}^{2}\right)^{1 / 2}
$$

avec :

$$
\begin{aligned}
& J_{a}=\left[\left(A-1 / a^{2}\right) E-D F\right] /[ D^{2}-\left(B-1 / a^{2}\right) \times \\
&\left.\times\left(A-1 / a^{2}\right)\right]
\end{aligned}
$$

et :

$$
\begin{aligned}
& K_{a}=\left[\left(B-1 / a^{2}\right) F-D E\right] / {\left[D^{2}-\left(B-1 / a^{2}\right) \times\right.} \\
&\left.\times\left(A-1 / a^{2}\right)\right]
\end{aligned}
$$

et des valeurs analogues pour $J_{b}$ et $K_{b}$ en remplaçant $a$ par $b$ dans (115) et (116).

Reste à calculer $\gamma_{1}$, la troisième valeur indépendante des neuf angles fixant la position de l'E.M.E. par rapport au champ. Il faut pour cela déterminer la projection $\left(X_{2}\right)_{a}$ de $a$ sur $X_{2}$ et donc extraire du système d'équations (111) la valeur de $X_{2}$, soit :

$$
\frac{X_{1}}{X_{2}}=\frac{D E-F\left(B-1 / r^{2}\right)}{D F-E\left(A-1 / r^{2}\right)}=L,
$$

soit :

$$
X_{1}^{2}=L^{2} X_{2}^{2}
$$

alors d'après (104) :

$$
X_{1}^{2}+X_{2}^{2}+X_{3}^{2}=r^{2}=X_{2}^{2}\left[1+L^{2}+\left(1 / J^{2}\right)\right]
$$

d'où

$$
X_{2}= \pm r /\left[1+L^{2}+\left(1 / J^{2}\right)\right]^{1 / 2}
$$

La projection $\left(X_{2}\right)_{a}$ de $a$ sur $X_{2}$ est donnée en remplaçant $r$ par $a$, d'où :

$$
a \cos \gamma_{1}= \pm a /\left[1+L_{a}^{2}+\left(1 / J_{a}^{2}\right)\right]^{1 / 2}
$$

soit :

$$
\cos \gamma_{1}= \pm 1 /\left[1+L_{a}^{2}+\left(1 / J_{a}^{2}\right)\right]^{1 / 2}
$$

avec :

$L_{a}=\left[D E-F\left(B-1 / a^{2}\right)\right] /\left[D F-E\left(A-1 / a^{2}\right)\right]$.

La valeur de $J_{a}$ est donnée par (115).

$N . B$. Dans le cas du champ uniaxial (traité en première partie), il suffit de déterminer $\alpha_{1}$ et $\alpha_{2}\left(\alpha_{3}\right.$ s'en déduisant à partir de $\sum \cos ^{2} \alpha_{i}=1$,

$i=1,2,3) . \quad \gamma_{1}$ n'est utile que dans le cas du champ triaxial.

Conclusion : développements et perspectives d'application.

La théorie présentée concerne les milieux biphasés, ou pouvant être considérés comme tels, par exemple les milieux poreux à une seule phase fluide. Dans les situations plus complexes, la théorie s'applique également dans le cadre d'une procédure itérative d'homogénéisation.

On aura noté que l'un des fondements de la théorie est l'uniformité du champ électrique dans l'inclusion. Autrement dit, la valeur du gradient est la même en tout point appartenant à l'inclusion. Il en est bien ainsi - on l'a vu - lorsque l'inclusion (homogène), plongée dans un milieu lui-même uniforme, est sphérique ou ellipsoïdale. Il y aurait lieu de rechercher si d'autre corps convexes fermés possèdent eux aussi cette propriété.

On sait par ailleurs qu'en physique macroscopique (Feynman 1969, Leontiev 1985), il y a une analogie formelle entre diverses lois de transfert d'énergie et d'écoulement de la matière. Dans tous les cas, le problème revient à traiter d'un champ qui obéit, au moins dans certains cas particuliers, aux mêmes équations différentielles générales : celles qui parmi les équations de Maxwell concernent les régimes indépendants du temps. Moyennant certaines précautions, il apparaît comme parfaitement fondé de mener l'étude générale des propriétés de champ par le biais de l'électrostatique ou par le choix de tout autre potentiel.

Enfin, nous reconnaissons bien volontiers qu'il reste encore à vérifier expérimentalement la validité de la théorie proposée pour le modèle de l'ellipsoïde quelconque. Il conviendra pour cela de mettre en jeu conjointement l'analyse d'images nécessaire à la description exacte du matériau et au calcul, et les mesures physiques sur des échantillons judicieusement choisis.

\section{Remerciements.}

Les auteurs ont plaisir à remercier M. le Professeur G. Ondracek et M.B. Ebel pour l'information généreusement accordée lors d'une visite (J.G.) à Karlsruhe en juin 1986 (et les discussions ultérieures); la Sté Nationale Elf-Aquitaine (Production) (MM. C. Jablon et V. Maury) puisque c'est lors du semestre sabbatique de l'un d'entre nous (J.G.) au Centre de Recherches de Pau que l'idée de ce travail fut esquissée ; Mme L. Brulé - sans elle l'obstacle de la langue serait resté infranchissable ; MM. J. Chapelle, J. Fronteau, M. Lepiller et G. Sabatier pour de nombreuses discussions.

\section{Annexe 1.}

Domaines de variation des coefficients $u, v, w$, des racines $t_{1}$ et $t_{2}$, de $A$ et de $(A-B+C+D)$.

Nous donnons ici, sans les démonstrations établies par nous-mêmes, le seul exposé des résultats. On rappelle que $0 \leqslant \lambda \leqslant 1$, de même pour $\mu, \nu, \ell, m$ et $n$. 
a) DOMAINE DE VARIATION DE

$$
\begin{aligned}
u=\ell(1-\mu) & (1-\nu)+m(1-\lambda) \times \\
& \times(1-\nu)+n(1-\lambda)(1-\mu) . \\
0 \leqslant \lambda \leqslant 1 \Rightarrow 0 & \leqslant \lambda-1 \leqslant 1 \Rightarrow \\
& \Rightarrow 0 \leqslant u \leqslant \ell+m+n \Rightarrow \quad 0 \leqslant u \leqslant 1
\end{aligned}
$$

avec $u=0$ pour $\ell=0$ et $\lambda=1$ (ou $m=0$ et $\mu=1$; ou $n=0$ et $\nu=1$ ); et $u=1$ pour $\ell=1$ et $\mu=\nu=0 \Leftrightarrow \ell=1$ et $\lambda=1$ (ou $m=\mu=1$; ou $n=\nu=1)$.

b) DOMAINE DE VARIATION DE

$$
w=\ell \mu \nu+m \lambda \nu+n \lambda \mu .
$$

La borne inférieure est évidemment nulle $(\ell=0$, $\lambda=0$ ).

La borne supérieure s'obtient en prenant $\lambda \leqslant \mu \leqslant \nu$ par exemple $\Rightarrow(\lambda-\mu) \leqslant 0$ et $(\lambda-\nu) \leqslant 0 \Rightarrow w=\mu \nu+m(\lambda-\mu) \nu+n(\lambda-\nu) \times$ $\mu \leqslant \mu \nu$, maximal pour $\mu=\nu$ d'où $2 \mu=1-\lambda$, donc $w \leqslant \mu^{2}$ ou $w \leqslant(1-\lambda)^{2 / 4} \Rightarrow 0 \leqslant w \leqslant 1 / 4$.

c) DOMAINE DE VARIATION DE

$$
v=1-u-w .
$$

La borne supérieure de $v$ est 1 pour $u=0$ $(n=0$ et $\nu=1)$ et $w=0 \quad(\ell=0, \lambda=0)$ soit $v=1$ pour $m=1$ et $\nu=1$.

La borne inférieure s'obtient en remarquant que $0 \leqslant \lambda^{2} \leqslant \lambda \leqslant 1$, de même pour $\mu$ et $\nu \Rightarrow$ d'après (23),

$$
\begin{aligned}
& v \geqslant \ell\left(\mu^{2}+\nu^{2}-2 \mu \nu\right)+ \\
& \quad+m\left(\lambda^{2}+\nu^{2}-2 \lambda \nu\right)+n\left(\lambda^{2}+\mu^{2}-2 \lambda \mu\right)
\end{aligned}
$$

soit $v \geqslant \ell(\mu-\nu)^{2}+m(\lambda-\nu)^{2}+n(\lambda-\mu)^{2} \geqslant 0$, borne inférieure de $v ; \quad 0 \leqslant v \leqslant 1$.

d) DOMAINES DE VARIATION DE $t_{1}$ et $t_{2}$ - $-t_{1} t_{2}=$ $w / u ; w$ et $u$ étant positifs, $t_{1}$ et $t_{2}$ sont de même signe; $t_{1}+t_{2}=-v / u ; v$ et $u$ étant positifs, $t_{1}$ et $t_{2}$ sont négatifs et, d'après (26), $\left|t_{2}\right| \geqslant\left|t_{1}\right|$.

(.) Pour $m=\mu=1, u=1$, soit d'après (51), $v=w=0 \Rightarrow t_{1}=t_{2}=0$.
(..) Pour $\ell=0$ et $\lambda=1$; on a $u=0$ et donc $t_{2} \rightarrow-\infty$.

(...) Pour $\ell=1$ et $\mu=\nu=1 / 2$, on a $u=w=1 / 4$ donc, d'après (51), $v=1 / 2$ d'où $T(t)=$ $\left(t^{2}+2 t+1\right) / 4=(t+1)^{2} / 4$, soit $t_{1}=t_{2}=-1$.

On a par ailleurs : $((1-\mu)(1-\nu)=$ $1-\mu-\nu+\mu \nu=\lambda+\mu \nu$ et deux identités analogues établies par permutation circulaire $\operatorname{sur} \lambda, \mu, \nu$, d'où

$$
\begin{aligned}
u=\ell(\lambda+\mu \nu)+m(\mu+\lambda \nu)+ \\
+n(\nu+\lambda \mu)=w+(\ell \lambda+m \mu+n \nu)
\end{aligned}
$$

soit $u \geqslant w$ et $t_{1} t_{2}=w / u \leqslant 1$; puisque $\left|t_{1}\right| \leqslant\left|t_{2}\right|$, on en déduit $\left|t_{1}\right|^{2} \leqslant 1 \Rightarrow-1 \leqslant t_{1} \leqslant 0$.

La borne -1 est atteinte pour $\ell=1$ et $\mu=\nu=1 / 2(\cdots)$.

La borne 0 est atteinte pour $m=\mu=1(\cdot)$.

D'autre part,

$$
-\infty<t_{2} \leqslant 0
$$

La borne $-\infty$ est atteinte pour $\ell=0 \quad$ et $\lambda=1(\cdots)$.

La borne 0 est atteinte pour $m=\mu=1(\cdot)$.

Le cas des racines doubles

$$
\text { et } \quad \begin{aligned}
t_{1} & =t_{2}=0(m=\mu=1) \\
t_{1} & =t_{2}=-1(\ell=1 \text { et } \mu=\nu=1 / 2)
\end{aligned}
$$

correspondent respectivement à des inclusions planes $(\lambda=\mu=0)$ et à des inclusions cylindriques $(\lambda=0)$; (cf. Eqs. (33a), (33b), et (33c).

e) Domaine DE VARIATION DE

$$
A=\frac{\lambda \mu \nu}{\ell \mu \nu+m \lambda \nu+n \lambda \mu} .
$$

On a démontré que $0 \leqslant A \leqslant 1$.

La borne 0 est atteinte pour $\lambda=0$ et/ou $\mu=0$ et/ou $\nu=0$.

La borne 1 est atteinte pour $n=1$ et $\lambda$ et $\mu$ très petits.

f) Domaine De VARIATION DE

$$
(A-B+C+D)=\frac{(\lambda-1)(\mu-1)(\nu-1)}{\ell(\mu-1)(\nu-1)+m(\lambda-1)(\nu-1)+n(\lambda-1)(\mu-1)}
$$

On a démontré que

$$
-1 \leqslant(A-B+C+D) \leqslant 0 .
$$

La borne 0 est atteinte pour $\lambda=1$ ou $\mu=1$ ou $\nu=1$.

La borne -1 est atteinte pour $n=1, \nu=0$ et $\lambda$ et $\mu$ très petits. 
Annexe 2.

Domaines de variation de $a_{1}, t_{1}^{\prime}, A$ et $C^{\prime}$ pour $\lambda=\mu$ (cas de l'ellipsoïde de révolution).

Les relations $\lambda=\mu, 0 \leqslant \nu \leqslant 1$ et $2 \lambda+\nu=1 \Rightarrow$ $0 \leqslant 1-2 \lambda \leqslant 1$ ou encore $0 \leqslant \lambda \leqslant 1 / 2 ; a_{1}, t_{1}^{\prime}, A$ et $C^{\prime}$ sont des fonctions de $n$ et de $\lambda$. Elles ont été étudiées graphiquement. On ne présente ici que les résultats.

\section{a) DOMAINE DE VARIATION DE}

$$
\begin{gathered}
a_{1}=n+(2-3 n) \lambda . \\
0 \leqslant a_{1} \leqslant 1
\end{gathered}
$$

avec $a_{1}=0$ pour $n=0$ et $\lambda=0$ et $a_{1}=1$ pour $\lambda=0$ et $n=1$ ou $\lambda=1 / 2$ et $n=0$.

\section{b) DOMAINE DE VARIATION DE}

$t_{1}^{\prime}=1-1 / a_{1}=1-1 /\{n+(2-3 n) \lambda\}$.

Pour

$$
0 \leqslant \lambda \leqslant 1 / 3, \quad-\infty<t_{1}^{\prime} \leqslant 0 .
$$

La valeur $-\infty$ est atteinte pour $n=0$ et $\lambda=0$. La valeur 0 est atteinte pour $n=1$ et $\lambda=0$. Pour

$$
1 / 3 \leqslant \lambda \leqslant 1 / 2, \quad-1 \leqslant t_{1}^{\prime} \leqslant 0 .
$$

La valeur -1 est atteinte pour $n=1$ et $\lambda=1 / 2$. La valeur 0 est atteinte pour $n=0$ et $\lambda=1 / 2$.

Rapprochant ces résultats de ceux de l'annexe 1 (cas général), on en déduit que :

$$
t_{1}^{\prime}=t_{2} \text { pour } 0 \leqslant \lambda \leqslant 1 / 3
$$

et

$$
C=0, \quad D=C^{\prime},
$$

et

$$
t_{1}^{\prime}=t_{1} \text { pour } 1 / 3 \leqslant \lambda \leqslant 1 / 2
$$

et

$$
C=C^{\prime}, \quad D=0
$$

\section{c) DOMAINE DE VARIATION DE}

$$
A=\frac{\lambda(1-2 \lambda)}{1-a_{1}}=\frac{\lambda(1-2 \lambda)}{1-n-(2-3 n) \lambda} .
$$

On démontre que : $\quad 0 \leqslant A \leqslant 1$

$$
\text { avec } \quad A=0 \text { pour } n=0 \text { et } \lambda=0
$$$$
\text { et } \quad A=1 \text { pour } n=1 \text { et } \lambda \rightarrow 0 \text {. }
$$

La fonction $A(n, \lambda)$ n'est en effet pas continue pour $n=1$ et $\lambda=0$. $A(1, \lambda)=1-2 \lambda$ et $A(n, 0)=0$ d'où $\lim _{\lambda \rightarrow 0} A(1, \lambda)=1$ et $\lim _{n \rightarrow 1} A(n, 0)=0$. De même $A(n, \lambda)$ n'est pas continue pour $n=0$ et $\lambda=1 / 2$, d'où

$$
\lim _{\lambda \rightarrow 1 / 2} A(0, \lambda)=1 / 2 \text { et } \lim _{n \rightarrow 0} A(n, 1 / 2)=0 .
$$

d) DOMAINE DE VARIATION DE

$$
C^{\prime}=1+\lambda \frac{a_{1}+2(\lambda-1)}{a_{1}\left(1-a_{1}\right)} ;
$$

avec $a_{1}=n+(2-3 n) \lambda$.

On démontre que $0 \leqslant C^{\prime} \leqslant 1$ pour $0 \leqslant \lambda \leqslant 1 / 3$ et $0 \leqslant C^{\prime} \leqslant 1 / 2$ pour $1 / 3 \leqslant \lambda \leqslant 1 / 2$, avec $C^{\prime}=0$ pour $\lambda=1 / 3$ et $C^{\prime}=1$ pour $\lambda=0$, $\forall n$.

Pour $n=0$ et $n=1$, on a $C^{\prime}=0$ pour $0 \leqslant \lambda \leqslant 1 / 2$. La fonction $C^{\prime}(n, \lambda)$ n'est pas continue pour $n=0$ et $\lambda=0$. $C^{\prime}(n, 0)=1$ et $C^{\prime}(0, \lambda)=0$. Elle n'est pas continue non plus pour $n=0$ et $\lambda=1 / 2$ : $\quad C^{\prime}(0, \lambda)=0 \quad$ et $\quad C^{\prime}(n, 1 / 2)=1-$ $1 /(2-n)$ d'où $\lim _{n \rightarrow 0} C^{\prime}(0,1 / 2)=1 / 2$.

e) CAlCul DES DiVERS COEFFICIENTS DANS LE CAS DE L'ELLIPSOÏDE DE RÉVOLUTION $(\lambda=\mu)$. - Portant dans (22) $\lambda=\mu$ (d'où $\nu=1-2 \lambda$ ) et utilisant $a_{1}=n+(2-3 n) \lambda$, on calcule : $u=(1-\lambda) a_{1}$; de même pour (23), $v=1-\left(a_{1}+\lambda\right)+2 \lambda a_{1}$; pour (24), $w=\lambda\left(1-a_{1}\right)$; pour (31), $q=\lambda^{2}(1-\lambda)$; pour (45), $r=\lambda(2-3 \lambda)$; et pour (46), $s=a_{1}+\lambda$.

Utilisant (50) et (51), on calcule le discriminant de $T(t)$, soit $\sqrt{\Delta}=(1-n)(1-3 \lambda)$ pour $0 \leqslant \lambda \leqslant 1 / 3$ et $\sqrt{\Delta}=-(1-n)(1-3 \lambda)$ pour $1 / 3 \leqslant \lambda \leqslant 1 / 2$ et donc $t_{1}=-\lambda a_{1} / u$ pour $0 \leqslant \lambda \leqslant 1 / 3$ et $t_{2}=-\lambda a_{1} / u$ pour $1 / 3 \leqslant \lambda \leqslant 1 / 2$.

Portant (24), (31), (45) et (46) dans (55) et (56), on a montré à l'aide du langage de calcul formel Numath que $c$ et $d$ peuvent se factoriser sous la forme :

$$
c=\left\{n(n-1)(1-\lambda)^{3}(1-3 \lambda)^{2}\right\} / u^{2}
$$

et

$$
d=\left\{n(n-1) \lambda(1-\lambda)^{2}(1-3 \lambda)^{2}\right\} / u^{2}
$$

de (43) et (44), on tire : $C=R_{1}\left(t_{1}\right)=\frac{c t_{1}+d}{a t_{1}+b}$, soit, pour $0 \leqslant \lambda \leqslant 1 / 3$ et

$$
\begin{aligned}
t_{1} & =-\lambda a_{1} / u, \\
C & =\frac{c\left(-\lambda a_{1}\right)+u d}{u\left(a t_{1}+b\right)} \\
& =\frac{u^{2} c\left(-\lambda a_{1}\right)+u^{3} d}{u^{3}\left(a t_{1}+b\right)}=0 .
\end{aligned}
$$


Les seuls coefficients qui interviennent dans la décomposition de $R(t)$ sont donc : $A, B$ et $D=C^{\prime}$.

Pour $1 / 3 \ll \lambda \leqslant 1 / 2$, on a $D=R_{1}\left(t_{2}\right)$, (45), avec $t_{2}=-\lambda a_{1} / u$; c'est donc $D$ qui est nul et $A, B$ et $C\left(=C^{\prime}\right)$ interviennent seuls dans la décomposition de $R(t)$.

\section{Annexe 3.}

Solution de l'équation générale pour des inlusions sphériques $(\lambda=\mu=\nu=1 / 3)$.

On vérifie, d'après (59) et (61) que, dans ces conditions, $A=1 / 3$ et, d'après (62), que $C^{\prime}=0$. L'équation (65) s'écrit alors :

$$
\frac{t^{1 / 3}}{|1-t|}=\frac{F\left(t_{n}\right)}{1-c_{D}}
$$

soit

$$
t=\frac{|1-t|^{3}\left\{F\left(t_{n}\right)\right\}^{3}}{\left(1-c_{D}\right)^{3}}
$$

Posant alors : $Q=\left(1-c_{D}\right) / F\left(t_{n}\right), u=1-t$, et $\varepsilon=$ signe de $(1-t)= \pm 1$, il vient $Q^{3}(1-u)=$ $\varepsilon u^{3}$, ou encore $: u^{3}+\varepsilon Q^{3} u-\varepsilon Q^{3}=0$; équation du $3^{\mathrm{e}}$ degré en $u$.

On rappelle que l'équation du $3^{\mathrm{e}}$ degré : $x^{3}+a x+b=0$ admet toujours la solution réelle :

$$
\begin{aligned}
x_{1}=\left\{(-b / 2)+\left\{\left(a^{3} / 27\right)+\left(b^{2} / 4\right)\right\}^{1 / 2}\right\}^{1 / 3}+ \\
+\left\{(-b / 2)-\left\{\left(a^{3} / 27\right)+\left(b^{2} / 4\right)\right\}^{1 / 2}\right\}^{1 / 3}
\end{aligned}
$$

les puissances fractionnaires portant sur des grandeurs considérées comme complexes.

Faisant alors $a=\varepsilon Q^{3}$ et $b=-\varepsilon Q^{3}$, on obtient la solution réelle :

$$
\begin{aligned}
u_{1}=\frac{Q}{2^{1 / 3}}\{[\varepsilon+\{1 & \left.\left.+\left(4 \varepsilon Q^{3} / 27\right)\right\}^{1 / 2}\right]^{1 / 3}+ \\
& \left.+\left[\varepsilon-\left\{1+\left(4 \varepsilon Q^{3} / 27\right)\right\}^{1 / 2}\right]^{1 / 3}\right\}
\end{aligned}
$$

Pour $\varepsilon=1$, la quantité $1+\left(4 \varepsilon Q^{3} / 27\right)$ est positive et l'équation $u^{3}+\varepsilon Q^{3} u-\varepsilon Q^{3}=0$ admet donc une seule racine réelle, et puisqu'on a montré que : $\frac{t^{1 / 3}}{|1-t|}=\frac{F\left(t_{\mathrm{n}}\right)}{1-c_{\mathrm{D}}}$ admet une solution unique, on a $t=1-u_{1}$.

Pour $\varepsilon=-1$, la quantité $1+\left(4 \varepsilon Q^{3} / 27\right)$ peut devenir négative, mais, par continuité, la solution réelle $t=1-u_{1}$ est bien toujours solution de

$$
\frac{t^{1 / 3}}{|1-t|}=\frac{F\left(t_{\mathrm{n}}\right)}{1-c_{\mathrm{D}}}
$$

On a vérifié le programme de résolution numérique de $F(t)=F\left(t_{\mathrm{n}}\right) /\left(1-c_{\mathrm{D}}\right)$ en comparant les résultats à la solution exacte $\left(1-u_{1}\right)$. Les résultats coïncident jusqu'à la cinquième décimale, au moins.

\section{Bibliographie}

EbEL B., Universität Karlsruhe, Diplomarbeit (1984).

Ebel B., Jeulin D., OndraceK G., The propertymicrostructure relationship of a composite consisting of five phases (MRS-Europe) 1985, pp. 187-194.

Feynman R. P., Leigton R. B., SAnds M., Le Cours de physique de Feynman (Bilingua AddisonWesley) 1969-1970.

Grolier J., Hucher M., Pouliquen J. M., Riss J., Prise en compte des données de la microscopie quantitative dans le calcul de la conductivité des roches. Généralisation de la théorie d'Ondracek, C.R. Acad. Sci. Paris, Série II 305 (1987) 14991502.

Gundersen H. J. G., Jensen E. B., Stereological estimation of the volume - weighted mean volume of arbitrary particles observed on random sections, J. Micros. 138 (1985) 127-142.

Hashin Z., Shtrikman S., A variational Approach to the Theory of the Effective Magnetic Permeability of Multiphase Materials, J. Appl. Phys. 33 (1962) 3125-3131.

Hashin Z., Assessment of the Self-Consistent Scheme
Approximation : Conductivity of particulate Composites, J. Compos. Mater. 2 (1968) 284300.

KLEIN P., ONDRACEK G., La corrélation entre structure et propriété de champ pour des solides frittés polyphasés. Rev. Chim. Miner. 18 (1981) 392411.

LÉontiev A., Théorie des échanges de chaleur et de masse (Ed. Mir, Moscou) 1985.

ONDRACEK G., The key positions of stereology in quantitative microstructure-correlations of multiphase materials, Acta Stereologica. Proc. 3rd Eur. Symp. Stereol. 2nd Part, 5-21 (1982).

ONDRACEK G., Zur quantitativen Gefüge-Feldeigenschafts-Korrelation mehrphasiger Werkstoffe, Teil I : Theorie des Gefugestruktur-Feldeigenschafts-Gleigungen zweiphasiger Werkstoffe, Metall, 1982, Heft 5, 36, 523 ; Teil II : Die Bestimmung des Gefügedaten durch quantitative Gefügeanalyse, Metall, Heft 12, 36, 1288 ; Teil III : Vergleich berechneter und experimenteller Feldeigenschaftswerte für metallische Zweiphasenwerkstoffe, Metall, Heft 10, 37, 1016 (1982-83). 
ONDRACEK G., The Quantitative Microstructure. Field Property Correlation of Multiphase and Porous Materials, Rev. Powder Metall. Phys. Ceram. 3 (1987) 205-322.

PeJsA R., Property Determination of Sintered Iron by Stereological Microstructure Analysis, Mikroskopie 37 (1980) 325-330.

RAMSAY J., Folding and fracturing of rocks (McGraw Hill) 1967.

Riss J., Average equivalent shape : classification of convex bodies. Intern. Congress Stereol., Caen, Acta Stereol., Suppl. III 6 (1987) 1034-1040.
Riss J., GroliEr J., Average equivalent shape : definition and identification. Acta Stereol. 5/1 (1986) 37-48.

STONER E. C., The Demagnetizing Factors for Ellipsoids (1945) 7, 36, 263, 803-821.

Weibel E. R., Stereological Methods. Practical Methods for Biological Morphometry (Academic Press) Vol. 1 (1979).

Stereological Methods. Theoretical Fondations (Academic Press) Vol. 2 (1980). 